\title{
Shh signaling guides spatial pathfinding of raphespinal tract axons by multidirectional repulsion
}

\author{
Lijuan Song ${ }^{1,2, *}$, Yuehui $\mathrm{Liu}^{1, *}$, Yang Yu${ }^{1, *}$, Xin Duan ${ }^{1}$, Shening $\mathrm{Qi}^{2}$, Yaobo Liu ${ }^{1}$ \\ ${ }^{I}$ State Key Laboratory of Brain and Cognitive Sciences, Institute of Biophysics, Chinese Academy of Sciences, 15 Datun Road, \\ Beijing 100101, China; ${ }^{2}$ Department of Histology and Embryology, School of Basic Medicine, Lanzhou University, Lanzhou \\ 730000, China
}

Relatively little is known about the molecular mechanisms underlying spatial pathfinding in the descending serotonergic raphespinal tract (RST) in the developing spinal cord, one of the most important nerve pathways for pain, sensory and motor functions. We provide evidence that ventral floor plate-secreted Sonic hedgehog (Shh) is responsible for the establishment of decreasing gradients in both the anterior-to-posterior (A-P) and the medialto-lateral (M-L) directions in the ventral spinal cord during serotonergic RST axon projection. Downstream components of the Shh pathway, Patched 1 (Ptch1) and Smoothened (Smo), were expressed in the serotonergic caudal raphe nuclei and enriched in the descending serotonergic RST axons. Diffusible Shh repulsion of serotonergic RST axons was shown to be mediated by Shh-Ptch1 interactions and derepression of Smo. Using a co-culture assay, we showed that A-P graded repulsion mediated by Shh signaling pushed the serotonergic axons caudally through the ventral spinal cord and M-L graded repulsion mediated by Shh signaling simultaneously restricted the serotonergic axons to the ventral and ventral-lateral funiculus. Prominent pathfinding errors of serotonergic RST axons were observed in various $S h h, P t c h 1$ and $S m o$ mutants. We conclude that Shh signaling-mediated multidirectional repulsion is required to push descending serotonergic RST axons in the A-P direction, and to restrict these axons to the ventral and ventral-lateral funiculus in the M-L direction. This is the first demonstration that Shh signalingmediated multidirectional repulsion of serotonergic RST axons maintains spatial axon pathfinding in the developing spinal cord.

Keywords: Shh; pathfinding; raphespinal tract; axon; repulsion

Cell Research (2012) 22:697-716. doi:10.1038/cr.2011.172; published online 8 November 2011

\section{Introduction}

The phenomenon of axon pathfinding is best explained by an axon guidance mechanism [1-3]. Recent findings have shown that several Wnt members and Sonic hedgehog (Shh) control axon projections along the anterior-to-posterior (A-P) axis of the developing spinal cord. In the mouse spinal cord, Wnt4-Frizzled 3 interactions attract commissural axons to ascend anteriorly [4], whereas Wnt5a/Wnt1-Ryk interactions

\footnotetext{
*These three authors contributed equally to this work. Correspondence: Yaobo Liu

Tel: + 86-10-64888559-815; Fax: + 86-10-64888559-802

E-mail: liuyaobo@sun5.ibp.ac.cn

Received 12 April 2011; revised 25 August 2011; accepted 26 September 2011; published online 8 November 2011
}

repel descending corticospinal axons posteriorly [5]. In the chick spinal cord, Shh also directly repels anterior projecting, midline commissural axons by interacting with Hip and it indirectly attracts these axons by regulating Wnt activity [6,7]. However, the molecular mechanisms controlling other axon projections in the developing spinal cord have not been investigated, particularly those guiding important descending nerve pathways, such as the raphespinal, rubrospinal, tectospinal and reticulospinal tracts [8-11]. Among these descending nerve tracks, the serotonergic projections of the brainstem raphe nuclei originate from the caudal raphe nuclei (CRN; B1-B3) and descend along the spinal cord, together constituting the serotonergic raphespinal tract (RST) $[12,13]$. A recent study has shown that Wnt5a attracts the descending serotonergic axons along the A-P direction in the mouse brainstem [14]. However, 
the molecular mechanisms underlying the guidance of the descending serotonergic RST in the mouse spinal cord have not been identified.

To investigate the molecular mechanisms underlying the guidance of the descending serotonergic RST in the mouse spinal cord, we studied the projection pattern of the serotonergic RST in mice. We found that serotonergic RST axons originate from the CRN beginning at stage E11.5. The pioneer axons of the serotonergic RST begin projecting to the spinal cord at E12.5. Between E12.5 and E16.5, these serotonergic RST axons continue to descend along the ipsilateral ventral spinal cord until they reach the caudal-most part of the spinal cord. All serotonergic RST axons are specifically restricted to the ventral and ventral-lateral funiculus as they descend through the ventral spinal cord.

Shh signaling components are involved in axon guidance [6, 15-18]. The canonical Shh signaling pathway is initiated by the binding of Shh to Patched (Ptch), a 12-pass transmembrane receptor. This binding relieves Ptch inhibition of Smoothened (Smo), a 7-pass transmembrane protein. Smo, which is also a G-protein-coupled-like receptor, initiates intracellular signal transduction that ultimately leads to growth cone movement $[16,17,19]$. Given the specific expression pattern of Shh in the floor plate and its subsequent diffusion into the ventral spinal cord [20, 21], we hypothesized that $\mathrm{Shh}$ is a potential axon guidance cue for descending serotonergic RST axons in the ventral spinal cord. Using in situ hybridization and immunostaining, we found that a high-to-low expression gradient of Shh was maintained in both the A-P and medial-to-lateral (M-L) directions during RST projection. Patched 1 (Ptch1) and Smo [22] were temporally expressed in serotonergic neurons of the $\mathrm{CRN}$ and enriched in the projecting serotonergic RST axons at different time points. In functional assays using Shh antibodies along with a Smo antagonist (SANT-1) [16] and Ptch $1^{4 l o o p 2}$-expressing serotonergic RST axons $[17,23]$, we demonstrated that both A-P and M-L, Shhdependent, graded repulsions of serotonergic RST axons were mediated by Shh-Ptch1 interactions and derepression of Smo. Prominent axon pathfinding errors of serotonergic RST axons have been observed in the A-P and $\mathrm{M}-\mathrm{L}$ directions in the spinal cord of various mutant mice such as Shh null mice [24], Sh $h^{\text {loxp } /}$ hypomorphic mice $[25,26]$, Ptch $1^{\text {sloop } 2}$ electroporated mice and NestinCre; Smo ${ }^{-/-}$mice [27-29].

Our evidence strongly supports that Shh signaling directly repels descending serotonergic RST axons posteriorly, and repulsively restricts these axons to the ventral and ventral-lateral funiculus by Shh-Ptch1 interactions and derepression of Smo. Therefore, Shhmediated multidirectional repulsion is required for the three-dimensional axon pathfinding of serotonergic RST axons in the developing spinal cord. Our findings expand our knowledge about axon guidance mechanisms in the mammalian spinal cord and identify other A-P guidance cues, in addition to the Wnt family. Moreover, our findings suggest a model in which a single axon guidance cue simultaneously provides multidirectional information for proper positioning of specific fiber tracts.

\section{Results}

Shh secreted from the floor plate establishes a decreasing $A-P$ gradient in the developing spinal cord

To explore the entire trajectory of serotonergic RST axons during development of the spinal cord, 5-hydroxytryptamine (5-HT) antibody immunostaining was applied to whole mounts and transverse sections of the spinal cord to label the projection of serotonergic RST axons. We found that serotonergic RST axons originated from stage E11.5 CRN and that pioneer axons initiated their projection into the spinal cord at E12.5 (Supplementary information, Figure S1A). These axons descended through the ventral spinal cord in the A-P direction until they reached the caudal end of the spinal cord at E16.5 (Supplementary information, Figure S1A, S1C, S1E and S1G). From E12.5 to E16.5, all descending serotonergic RST axons were restricted specifically inside the ventral spinal cord, including the ventral and ventral-lateral funiculus (Supplementary information, Figure S1B, S1D, S1F and S1H). Axonal branching into the gray matter of the ventral spinal cord was observed at E16.5, the same time as the RST completed its projection through the spinal cord (Supplementary information, Figure $\mathrm{S} 1 \mathrm{H})$.

Shh is strongly expressed in the floor plate during early embryonic stages and diffuses into the ventral spinal cord to form a ventral-dorsal decreasing gradient $[15,25,30]$. Here, we investigated Shh expression patterns along the A-P axis of the spinal cord. At E12.5, descending serotonergic RST axons began projecting into the spinal cord, where the gradient of the guidance molecule(s) is thought to be established prior to axon arrival. The region between the upper cervical and thoracic spinal cord was dissected out and used to prepare a horizontal section. Serial transverse sections were cut at $800-\mu \mathrm{m}$ intervals every section along the A-P axis. In situ hybridization revealed a high-to-low gradient of Shh mRNA along the A-P axis of the E12.5 spinal cord (Figure 1A). Quantification of the relative density of signals provided further evidence of this gradient in the 


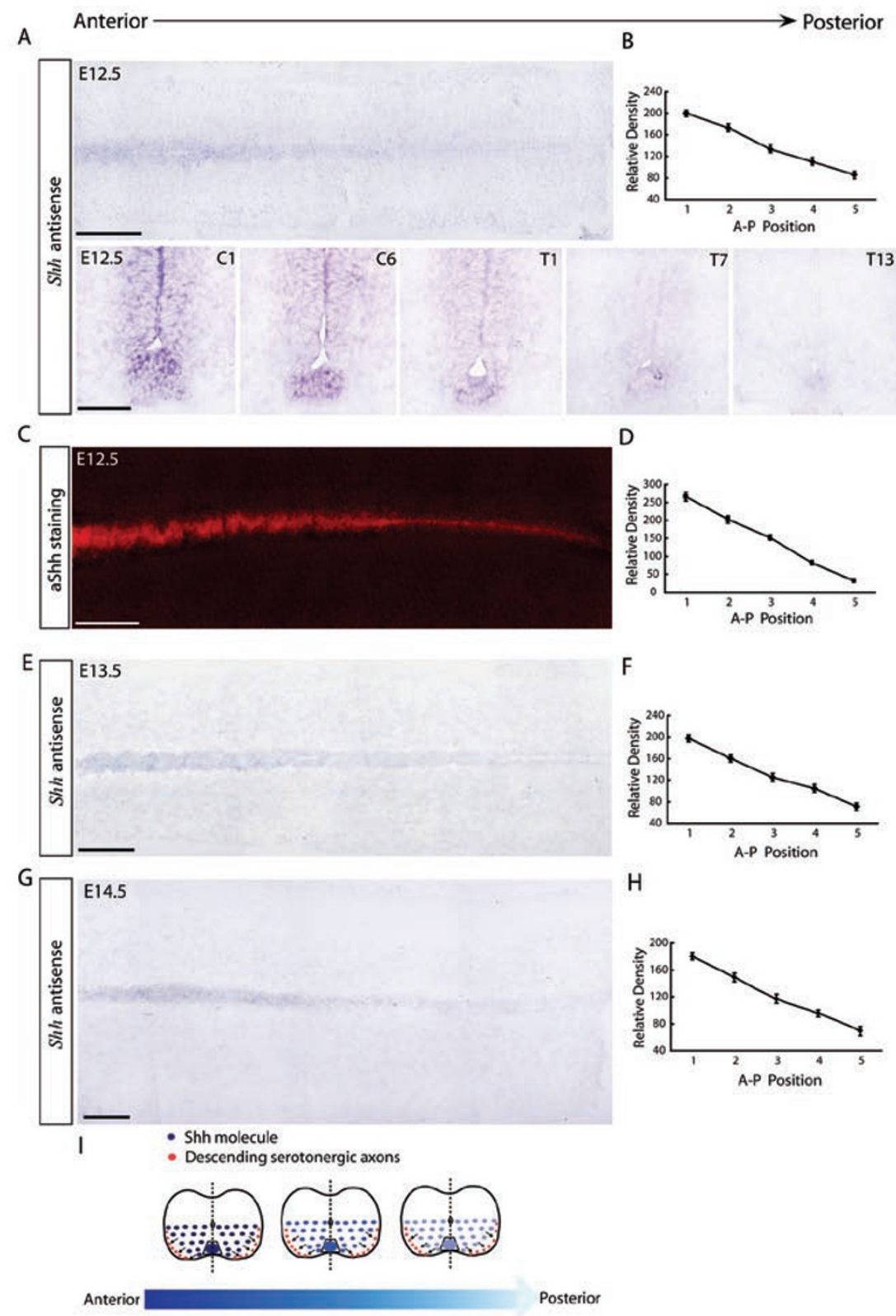

Figure 1 Shh secreted from the floor plate establishes a decreasing A-P gradient in the developing spinal cord. (A) A 4-5 mm, decreasing A-P gradient of Shh mRNA was detected in the floor plate of E12.5 spinal cords. In situ hybridization with an Shh antisense probe was conducted on one horizontal and a series of coronal sections at intervals of $800 \mu \mathrm{m}$. Scale bars: top 500 $\mu \mathrm{m}$; bottom $50 \mu \mathrm{m}$. (B) Quantification of positive signals showed a decreasing A-P gradient of Shh mRNA in the E12.5 spinal cord. (C) Shh secreted from the floor plate formed a $4-5 \mathrm{~mm}$, decreasing A-P gradient in the E12.5 spinal cord. Immunostaining with anti-Shh was conducted using one horizontal section. Scale bar: $500 \mu \mathrm{m}$. (D) Quantification of positive signals showed a high-to-low gradient of Shh protein in the A-P direction of E12.5 spinal cords. (E) The decreasing A-P gradient of Shh mRNA was maintained in the floor plate of E13.5 spinal cords. In situ hybridization with an Shh antisense probe was conducted using horizontal sections. Scale bar: $800 \mu \mathrm{m}$. (F) Quantification of the positive signals in C showed a sustained, decreasing A-P gradient of Shh mRNA in E13.5 spinal cords. (G) The decreasing A-P gradient of Shh mRNA was maintained in the floor plate of E14.5 spinal cords. In situ hybridization with an Shh antisense probe was conducted on horizontal sections. Scale bar: $1000 \mu \mathrm{m}$. (H) Quantification of the positive signals in E showed a sustained, decreasing A-P gradient of Shh mRNA in E14.5 spinal cords. The relative density of the positive signal was measured by Image $\mathrm{J}$ software (NIH). The relative density of each point was averaged from at least three different spinal cords and statistical analysis was performed using the Student's $t$-test. Results are presented as the mean \pm S.E.M. (I) Schematic diagram explaining our hypothesis that an Shh gradient directs the projection of serotonergic RST axons. 
cervical and thoracic spinal cord (Figure 1B). Similarly, immunostaining with anti-Shh revealed a high-to-low gradient of Shh protein along the A-P axis of the E12.5 spinal cord (Figure 1C and 1D). Serotonergic RST axons reached the thoracic spinal cord at E13.5 (Supplementary information, Figure S1C) and reached the lumbar spinal cord at E14.5 (Supplementary information, Figure S1E). At E13.5 and E14.5, we found that the A-P Shh mRNA gradient was extended and fully covered the continuous projection of descending serotonergic axons along the A-P axis of the developing spinal cord (Figure 1E, 1F, $1 \mathrm{G}$ and $1 \mathrm{H}$ ). It thus seemed likely that the pathfinding of these axons was directed by a high-to-low Shh gradient.

In summary, we hypothesized that in addition to Shh diffusion in the ventral spinal cord, Shh also establishes a gradient along the A-P axis of the ventral spinal cord, coincident with the pathway along which serotonergic RST axons descend (Figure 1I). Serotonergic RST axons arrive at the cervical spinal cord at E12.5. At the same time, a decreasing A-P Shh gradient is set up along the projecting pathway and may repulsively guide these axons to project posteriorly during later stages of spinal cord development (Figure 1I). In addition, this repulsion may also restrict the RST axons to the ventral and ventral-lateral funiculus as a result of Shh diffusion from the floor plate, which establishes a decreasing gradient from the midline to the lateral edge of the ventral spinal cord (Figure 1I).

\section{Ptch1 and Smo are expressed in CRN neurons and descending RST axons}

Because Shh is a potential repellent that may guide descending serotonergic axons along the ventral spinal cord, we also examined the expression pattern of downstream components of the Shh signaling pathway, including Ptch1 and Smo. We postulated that Ptch1 and Smo are expressed in serotonergic neurons of the CRN and transported to the growth cone of projecting RST axons. Ptch1 may, therefore, interact with diffusible Shh, which is expressed in the ventral spinal cord, to relieve Ptch1 inhibition of Smo. Using in situ hybridization, Ptch1 and Smo were found to be strongly expressed in the ventral side of the E12.5 caudal brainstem, which contains the serotonergic CRN (Figure 2B and 2C). This temporal expression pattern of Ptch1 and Smo correlated with the initiation of serotonergic RST axon projection into the E12.5 spinal cord, indicating that Ptch1 and Smo are the critical downstream molecules mediating the Shh guidance signaling beginning at stage E12.5.

If Shh signaling directly guides serotonergic axon pathfinding through the ventral spinal cord, then Ptch1 and Smo expression should be maintained in the projecting axons coincident with the sustained Shh gradient. To confirm this hypothesis, we conducted co-immunostaining of the E12.5 caudal brainstem containing the $\mathrm{CRN}$ and pioneer axons of the descending serotonergic RST. We found that both Ptch1 and Smo were expressed specifically in serotonergic neurons of the E12.5 CRN and pioneer serotonergic RST axons (Figure 2D and 2E). Ptch 1 staining was eliminated by preincubation of the Ptch 1 antibody with $10 \mu \mathrm{g} /$ $\mathrm{ml}$ of recombinant Ptch1 (Supplementary information, Figure S2A). Smo staining was absent from the tissue from a Nestin-Cre; $\mathrm{Smo}^{-/-}$mouse (Supplementary information, Figure S2B). The specific immunostaining patterns of Ptch1 and Smo in serotonergic neurons are shown in high-magnification images in Supplementary information, Figure S3. These results demonstrated the specificity of the Ptch1 and Smo antibody staining. Coimmunostaining of coronal sections of E14.5 spinal cord was conducted to investigate Ptch1 and Smo expression in the projecting axons of serotonergic RST axons. Expression of Ptch1 and Smo was enriched in descending axons of the serotonergic RST during the projection period (Figure $2 \mathrm{G}$ and $2 \mathrm{H}$ ). Therefore, based on the expression pattern of Shh, Ptch1 and Smo, we concluded that Shh signaling, via repulsion, probably directs serotonergic RST axons along the A-P axis of the spinal cord. Moreover, Shh repulsion further restricts these axons to the ventral and ventral-lateral funiculus in the M-L direction.

\section{Diffusible Shh protein repels serotonergic RST axons via Ptch1 and Smo}

To verify the repulsion of serotonergic RST axons by diffusible Shh, we identified the area in the CRN where serotonergic RST axons originate at E12.5 by wholemount immunostaining with anti-5-HT (Figure 3A). E12.5 CRN explants were subsequently co-cultured with COS1 cell aggregates transfected with an Shh expression construct or an empty vector. Shh expression in transfected COS1 cells was examined by immunostaining using anti-Shh (Supplementary information, Figure S4). Serotonergic RST axons growing from E12.5 CRN explants were repelled strongly by Shh-expressing COS1 cell aggregates, whereas serotonergic RST axons showed no directional response to control vector-transfected aggregates (Figure 3B).

Bearing in mind the temporal expression patterns of Shh, Ptch1 and Smo when the serotonergic RST axons are projecting, we used different inhibitors of the Shh signaling pathway to identify the signaling components mediating the repulsion of serotonergic RST axons. To examine how Shh may regulate the response of 

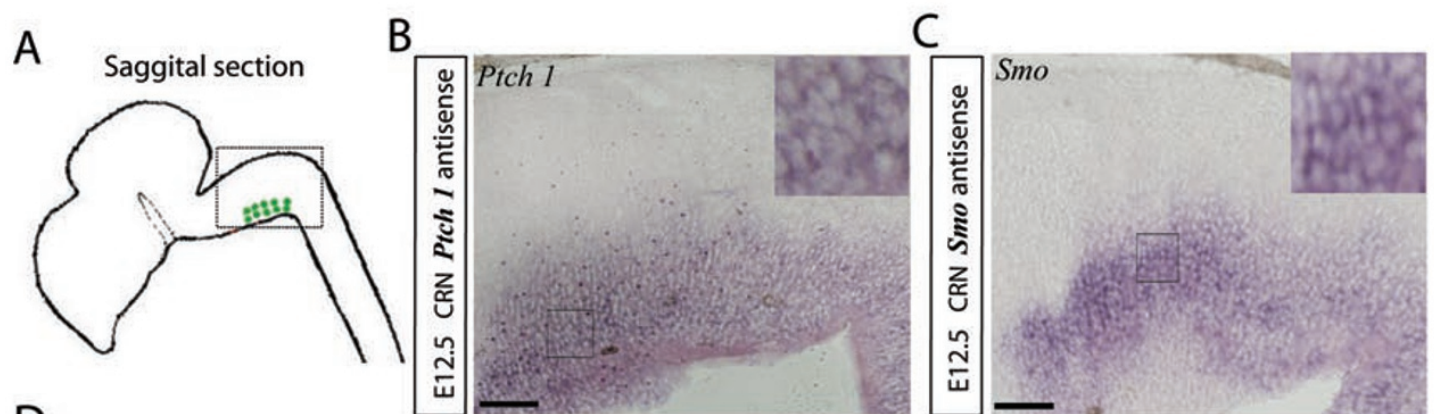

$\mathrm{D}$
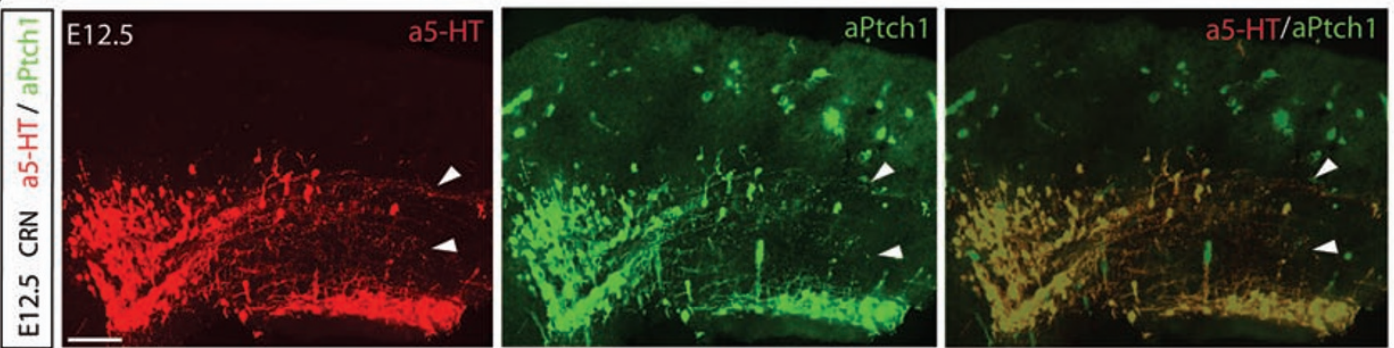

$\mathrm{E}$
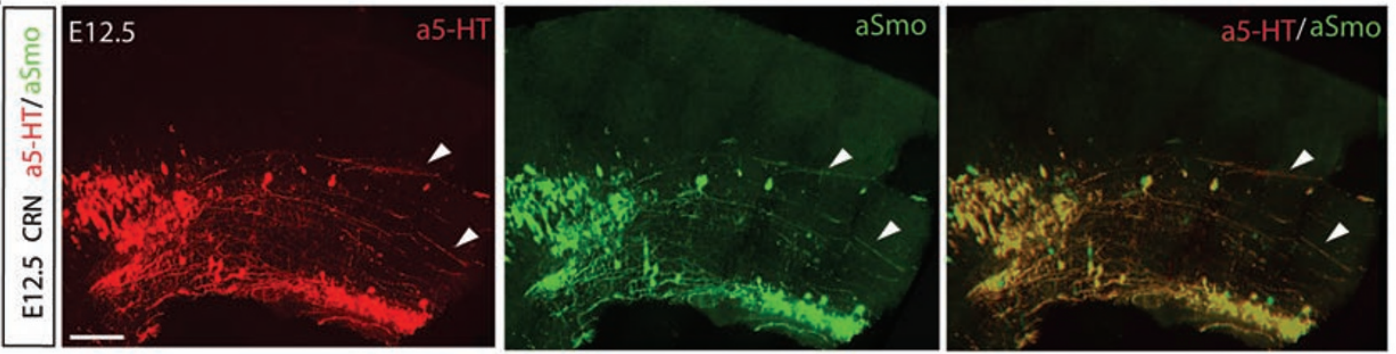

$\mathrm{F}$
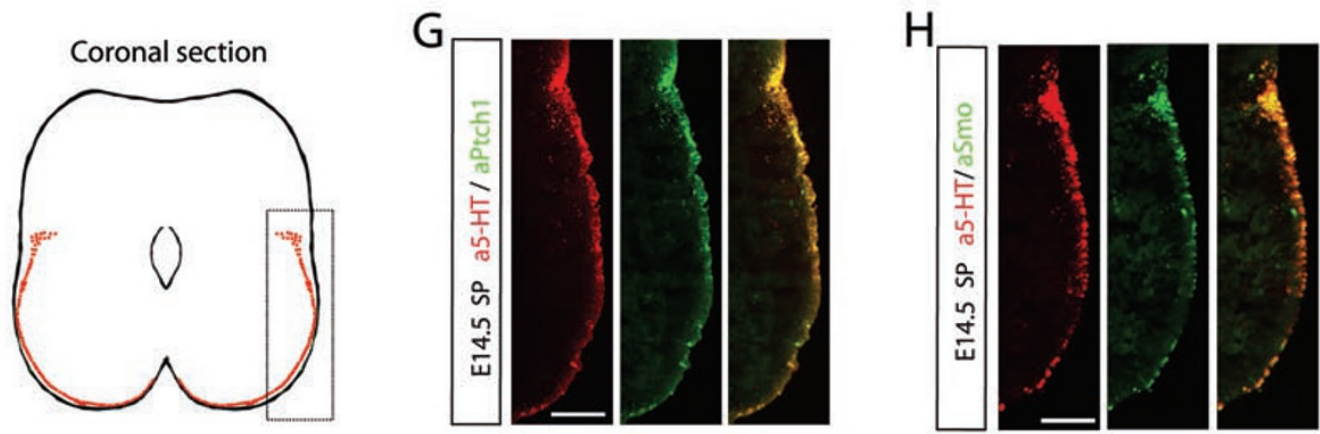

Figure 2 Ptch1 and Smo are temporally expressed in CRN neurons and descending RST axons. The caudal brainstem containing the CRN and pioneer RST axons (A) was cut into sagittal sections (shown in the rectangle). Sagittal sections were used to conduct in situ hybridization and co-immunostaining. At stage E12.5, Ptch1 (B) and Smo (C) mRNA was expressed in the CRN neurons. High-magnification images of selected areas are shown in insets (B, C). Ptch1 (D) and Smo (E) proteins were strongly expressed in serotonergic neurons of the E12.5 CRN and also enriched in the serotonergic RST axons originating from the E12.5 CRN. The spinal cord was cut into coronal sections (F) and descending RST axons (shown in the rectangle) in the spinal cord were co-immunostained with anti-5-HT and anti-Smo or anti-5-HT and anti-Ptch1. At stage E14.5, Ptch1 $(\mathbf{G})$ and Smo $(\mathbf{H})$ proteins were highly enriched in the projecting RST axons in the spinal cord. Scale bar: $100 \mu \mathrm{m}$. White arrowheads point to serotonergic RST axons.

serotonergic RST axons to repulsive guidance cues and their pathfinding in the spinal cord, we first used ex utero electroporation to test the function of the Shh receptor, Ptch1, by expressing a construct encoding a dominant-negative Ptch1 in serotonergic RST axons. We used a deleted form of Ptch1, Ptch1 $1^{\text {4loop } 2}$, which 
lacks the Shh-binding domain in its second extracellular loop, making it a constitutive inhibitor of Smo [17, 18, 23]. Ptch $1^{\text {4loop } 2}$ was cloned into the IRES-pCIG2 vector to allow expression in neurons and visualization of neurons expressing Ptch1 $1^{\text {4loop } 2}$ with enhanced green fluorescence protein (EGFP). Ptch1 $1^{\text {4loop } 2}$ was expressed in serotonergic axons growing from $\mathrm{CRN}$ explants by $e x$ utero electroporation (Figure 3A). Ptch1 $1^{\text {4loop } 2}$-expressing
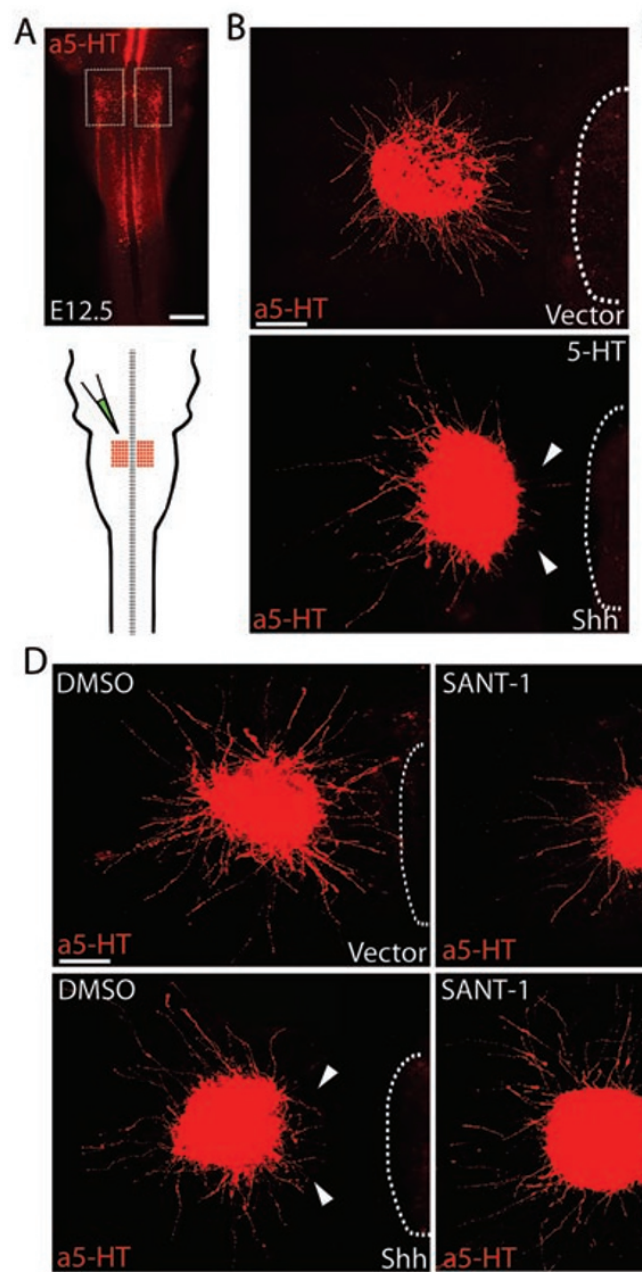


E
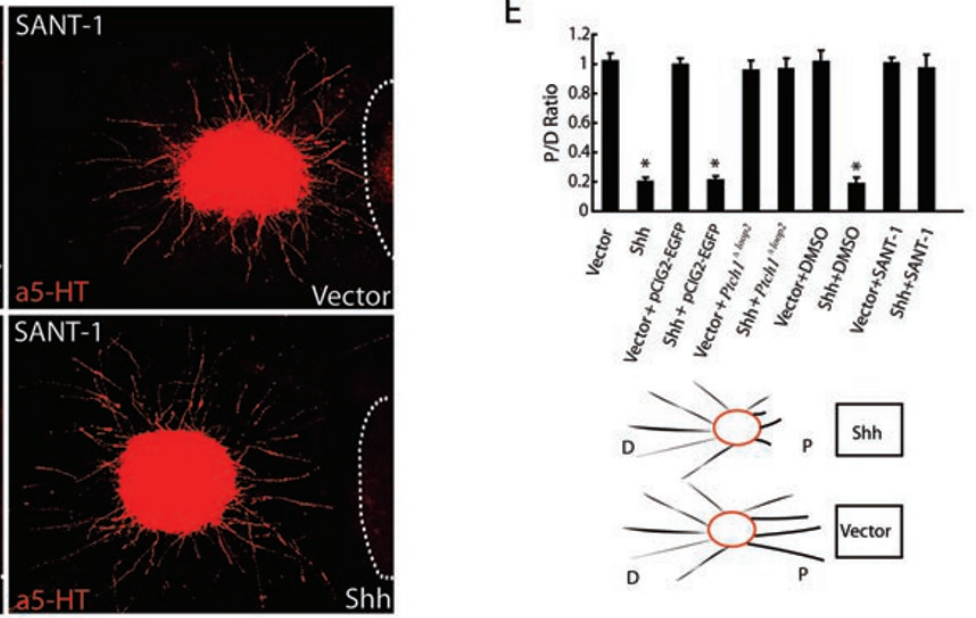

Figure 3 Diffused Shh repels serotonergic RST axons via Ptch1 and Smo. E12.5 CRN explants were co-cultured with COS1 cell aggregates transfected with empty vectors or Shh expression constructs. (A) Ex utero electroporation of the E12.5 mouse CRN. Whole-mount immunostaining with anti-5-HT was used to identify the E12.5 CRN, from which the serotonergic RST axons originated (shown in the rectangle and the red area of the brainstem diagram). Scale bar: $500 \mu \mathrm{m}$. (B) Secreted Shh strongly repelled serotonergic RST axons growing out from the CRN explants. (C) Shh-Ptch1 interactions mediate Shh repulsion of projecting RST axons. Secreted Shh strongly repelled serotonergic RST axons growing from CRN explants that expressed only EGFP. By comparison, secreted Shh had no repulsive effect on serotonergic RST axons expressing Ptch $1^{\text {Nloop2 }}$. White arrowheads indicate deflected axons. White dashed lines outline the boundary of cell aggregates. Scale bar: $200 \mu \mathrm{m}$. (D) Derepression of Smo mediates Shh repulsion of projecting RST axons. Secreted Shh strongly repelled serotonergic RST axons growing from the CRN explants. This repulsion was effectively interrupted by adding SANT-1. White arrowheads indicate deflected axons. White dashed lines outline the boundary of cell aggregates. Scale bar: $200 \mu \mathrm{m}$. (E) Quantification of axon outgrowth in the cell aggregate assay. The repulsion of serotonergic RST axons caused by secreted Shh was mediated by Ptch 1 and Smo receptors. The P/D ratio was taken as a measure of axon outgrowth. The experiment was replicated at least five times. Statistical analysis was performed using the Student's $t$-test. Results are presented as the mean \pm S.E.M. $(n$ $\geq 5)$. Asterisks indicate values that differ significantly from values in the control groups $(P<0.05)$. Vector group: $1.018 \pm 0.056$; Shh group: $0.198 \pm 0.078$; Vector + pCIG2 group: $0.993 \pm 0.048$; Shh + pClG2 group: $0.209 \pm 0.029$; Vector + Ptch1 $1^{\text {sloop } 2}$ group: $0.958 \pm 0.065$; Shh + Ptch $1^{\Delta l o o p 2}$ group: $0.966 \pm 0.072 ;$ Vector + DMSO group: $1.013 \pm 0.082 ;$ Shh + DMSO group: 0.186 \pm 0.045 ; Vector + SANT-1 group: $1.006 \pm 0.038$; Shh + SANT-1 group: $0.986 \pm 0.091$. 
axons showed no detectable response to Shh protein secreted from COS1 cell aggregates (Figure 3C). The specific co-immunostaining pattern of EGFP and 5-HT in the axons is shown in high-magnification images in Supplementary information, Figure S5. Ptch1 $1^{\text {4loop2 }}$ blocked Shh-dependent axon repulsion, indicating that interactions between Shh and Ptch1 are required to activate this repulsion.

We also used this robust in vitro assay to further assess the molecular mechanism of axon guidance by Shh. We inhibited Smo by the addition of SANT-1 [16], an antagonist of the Shh pathway that binds Smo directly. Addition of SANT-1 to the culture system blocked Shh repulsion, and no differences in axon orientation were observed compared to control cultures (Figure 3D). The fact that SANT-1 blocked Shh-dependent axon repulsion indicated that derepression of Smo is required to activate the repulsion.

Quantification of axon growth was performed by calculating the axon proximal/distal (P/D) ratio from at least five experimental replicates. Shh repulsion was significantly blocked by Ptch $1^{\text {sloop } 2}$ or SANT-1 (Figure $3 \mathrm{E})$. In this functional assay, there were no significant fluctuations in the average axon length in any of the experimental groups. These results indicated that diffusible Shh protein repels serotonergic RST axons growing from the E12.5 CRN in vitro and that this repulsion is mediated by Shh signaling. Therefore, we deduced that, in the A-P direction, graded repulsion directs the serotonergic RST axons caudally through the ventral spinal cord. Moreover, because this repulsion may also exist in the M-L direction in the ventral spinal cord, Shh may also be capable of simultaneously restricting these axons in the direction of the ventral and ventral-lateral funiculus.

\section{A-P graded repulsion is mediated by Shh signaling}

To verify our hypothesis that serotonergic RST axons are guided posteriorly by an A-P graded repulsion via Shh signaling, we established a functional assay, the A-P graded assay. This assay allows for observation of the effects of the ventral spinal cord Shh gradient on descending RST axons. In this assay, we placed three E12.5 CRN explants at different points along the A-P axis, facing the ventral side of the ipsilateral spinal cord (Figure 4A), which was expected to secrete diffusible axon guidance cues and to direct the serotonergic RST axons. The CRN explants and ventral spinal cord then were co-cultured for $60 \mathrm{~h}$ in a collagen system. Wholeculture immunostaining with anti-5-HT was used to confirm that serotonergic RST axons were growing from the CRN explants. We found that the entire ventral spinal cord displayed an A-P graded repulsion toward serotonergic RST axons growing from the CRN explants, with the anterior spinal cord displaying a stronger effect than the posterior spinal cord (Figure 4B). This finding provided further evidence that a diffusible axon guidance cue from the ventral spinal cord establishes a high-to-low repulsive gradient and directs serotonergic RST axons to project into the caudal segment of the ventral spinal cord.

We used an Shh blocking antibody (5E1), constitutive inhibition of Ptch1 (Ptch1 $1^{\text {4loop2 }}$ ex utero electroporation) and SANT-1 to effectively block A-P graded repulsion in the ventral spinal cord (Figure 4B). The specific, axonal, co-immunostaining pattern of EGFP and 5-HT is shown in high-magnification images in Supplementary information, Figure S6. The Shh antibody showed a weaker inhibitory effect than either SANT-1 or Ptch $1^{\text {4loop2 }}$ ex utero electroporation, as ligand-targeted inhibitors usually are less efficient than receptor-targeted inhibitors. The P/D ratios of axon outgrowth differed significantly between experimental groups, which further verified our hypothesis (Figure 4C). We concluded that the A-P repulsion guiding the posterior projection of serotonergic RST axons is produced by the ventral spinal cord and reflects diffusible Shh protein originating from the floor plate and establishing a strong-to-weak gradient along the A-P axis of the ventral spinal cord. The repulsion of serotonergic RST axons is mediated by Shh binding to Ptch1 and subsequent derepression of Smo.

\section{$M-L$ graded repulsion is mediated by Shh signaling}

The above data showed that Shh signaling guides descending serotonergic RST axons projecting posteriorly by a decreasing A-P repulsion gradient. We also noted that posteriorly projecting serotonergic RST axons are tightly restricted to the ventral and ventrallateral funiculus when they are descending through the ventral spinal cord. It is well established that Shh diffuses through the entire ventral spinal cord. Therefore, it is likely that Shh guides the pathfinding of serotonergic RST axons by multidirectional repulsion, where Shh directs axons in the A-P direction and simultaneously restricts them in the M-L direction to the ventral and ventral-lateral funiculus. To verify this hypothesis, E12.5 CRN explants were dissected and placed at the lateral side of slices dissected from the ipsilateral spinal cord (Supplementary information, Figure S7A) before co-culture in a collagen system. Whole-culture immunostaining with anti-5-HT was used to confirm that serotonergic RST axons originated from the CRN explants. We found that a diffusible repellent was secreted from the ventral spinal cord in an M-L direction and repelled serotonergic RST axons from the E12.5 
CRN (Supplementary information, Figure S7B). As expected, this repulsion was blocked effectively by the addition of either anti-Shh, SANT-1 or the expression of Ptch $^{1 \text { lloop } 2}$ in the serotonergic axons growing from the E12.5 CRN explants. However, the blocking efficiency of anti-Shh was slightly less than that of SANT-1 or Ptch $1^{\text {Sloop2 }}$ (Supplementary information, Figure S7B). The $\mathrm{P} / \mathrm{D}$ ratios of axon outgrowth differed significantly between groups, which demonstrated that repellent Shh signaling is also in the M-L direction, and restricts

A
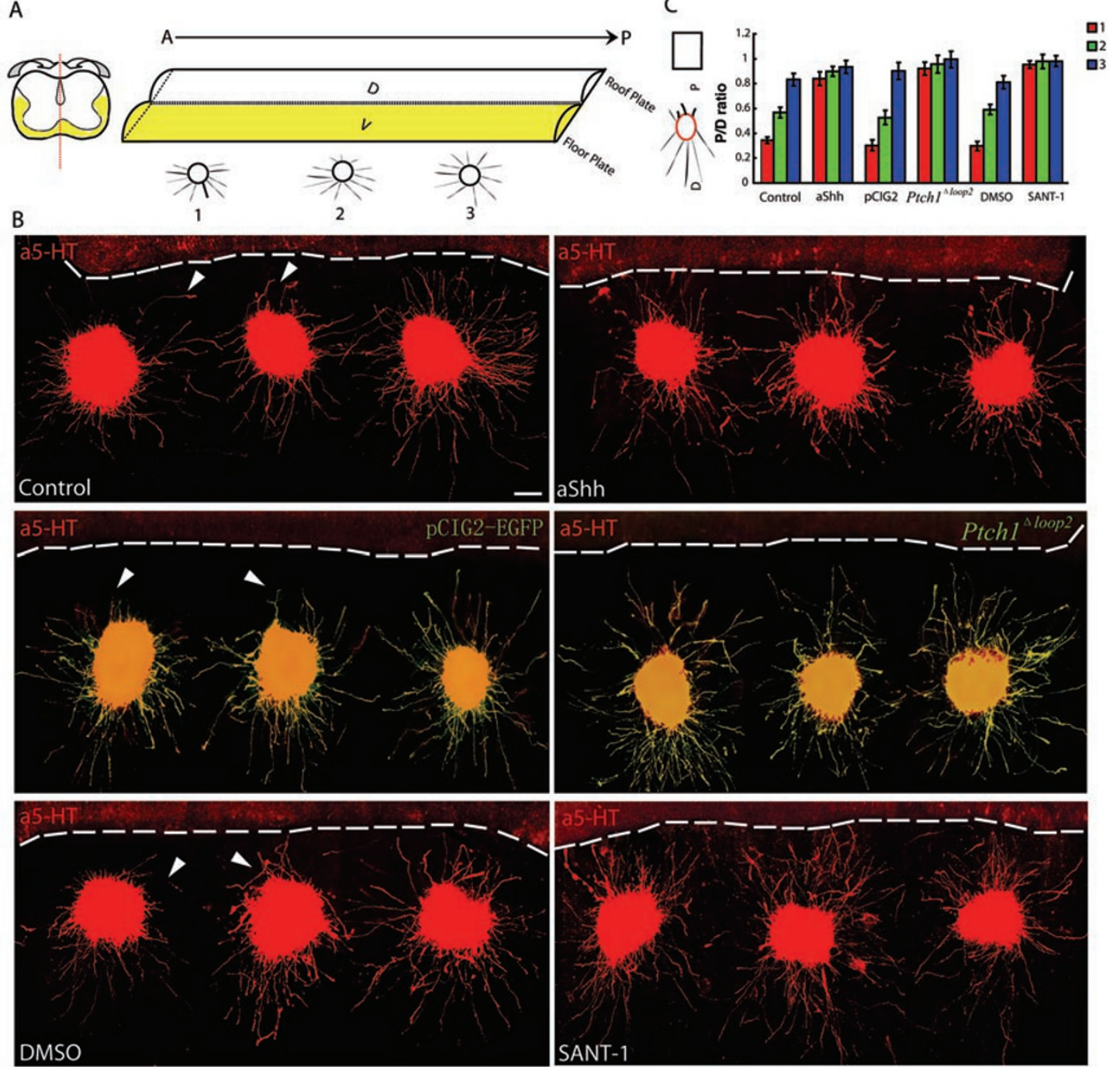

Figure 4 A-P graded repulsion is mediated by Shh signaling. (A) Schematic depiction of the A-P graded assay. After dissecting the ipsilateral CRN and spinal cord of E12.5 embryos, three CRN explants were placed at intervals along the ventral spinal cord in the A-P direction. After culture, immunostaining with anti-5-HT was used to confirm that descending serotonergic RST axons were growing from the CRN explants. (B) Decreasing A-P repulsion of serotonergic RST axons was detected clearly along the ventral spinal cord in the A-P assay. This graded repulsion was abolished effectively when the Shh antibody (5E1) or SANT-1 was added to the co-culture system or when Ptch $1^{\Delta / \mathrm{lop} 2}$ was expressed in serotonergic RST axons by coculturing the Ptch $1^{\Delta / o o p 2}$-electroporated CRN with the ventral spinal cord. White arrowheads indicate deflected axons. White dashed lines outline the boundary of the ventral spinal cord. Scale bar: $100 \mu \mathrm{m}$. (C) Quantification of axon outgrowth in the A-P assay. Axon outgrowth was measured using the P/D ratio. A-P graded repulsion was abolished after the Shh signaling components were blocked individually. The experiment was replicated at least five times. Statistical analysis was performed using the Student's $t$-test. Results are presented as the mean \pm S.E.M. Control group: $0.345 \pm 0.026 ; 0.568 \pm 0.042 ; 0.832 \pm$ 0.049; Shh Antibody group: $0.839 \pm 0.055 ; 0.898 \pm 0.040 ; 0.933 \pm 0.052 ;$ pCIG2-EGFP group: $0.303 \pm 0.043 ; 0.527 \pm 0.057$; $0.902 \pm 0.069$; Ptch $1^{\text {\loop } 2}$ group: $0.921 \pm 0.052 ; 0.956 \pm 0.071 ; 0.995 \pm 0.066$; DMSO group: $0.297 \pm 0.037 ; 0.591 \pm 0.041 ; 0.811$ \pm 0.053 ; SANT-1 group: $0.954 \pm 0.029 ; 0.979 \pm 0.057 ; 0.981 \pm 0.045$. 
the descending axons to the ventral and ventral-lateral funiculus (Supplementary information, Figure S7C).

Immunostaining for Shh revealed highly concentrated Shh expression in the floor plate of the E12.5 spinal cord, and Shh decreased gradually in the M-L direction from the floor plate (Figure 5A). Quantification of the relative intensity of Shh immunostaining in the ventral spinal cord showed a decreasing distribution of Shh in the M-L direction (Figure 5B and 5C). These data strongly suggested that graded Shh concentrations repel the serotonergic axons in the M-L direction, and restrict them to the ventral and ventral-lateral funiculus.

To further verify that serotonergic RST axons are guided by an M-L graded repulsion via Shh signaling, we also established a functional assay, the M-L graded assay. In this assay, we cut out three explants from the
A

B

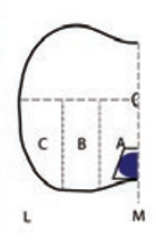



$\mathrm{E}$
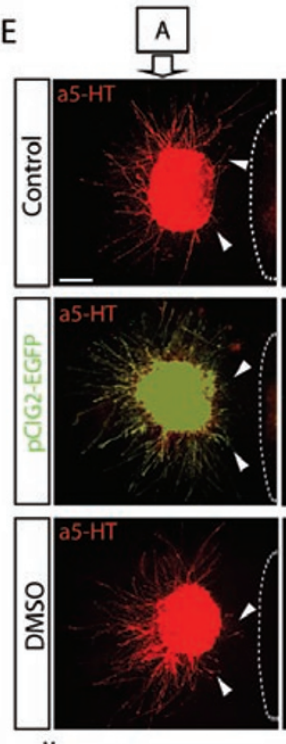
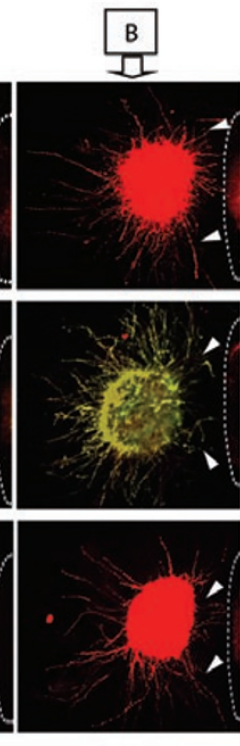

C

$\mathrm{D}$

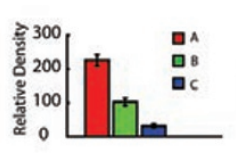

D - Shh molecule - Descending serotonergic axons

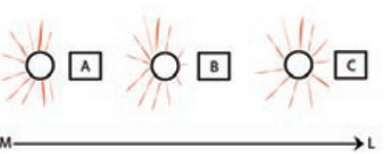

$\mathrm{F}$

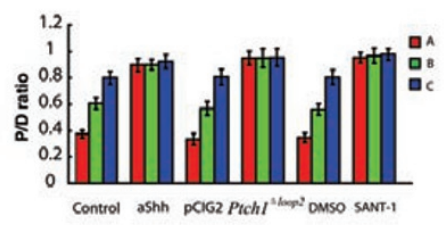

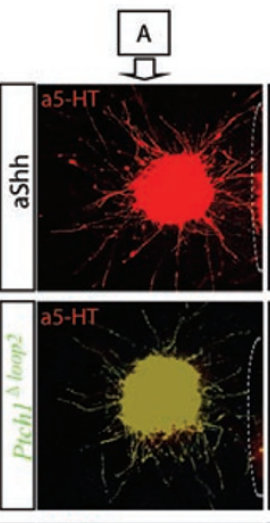
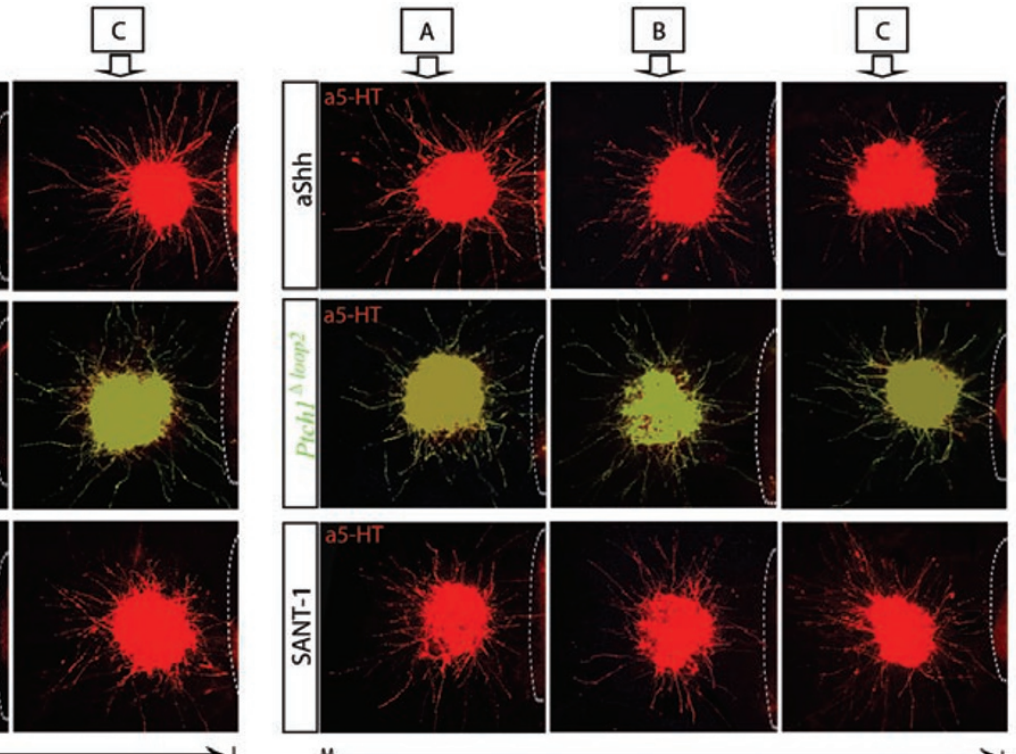

Figure $5 \mathrm{M}$-L graded repulsion is mediated by Shh signaling. (A) The decreasing Shh gradient in the M-L direction was shown by immunostaining for Shh in coronal sections of the E12.5 upper cervical spinal cord (outlined by the dashed line). Scale bar: $200 \mu \mathrm{m}$. (B) The ventral spinal cord is outlined as part A, B and C in the M-L direction. (C) Quantification of positive immunostaining signals in the $A, B$ and $C$ areas showed a decreasing $M-L$ gradient of Shh in the E12.5 ventral spinal cord. (D) Schematic depiction of the M-L graded assay. Three explants (A, B and C) were dissected from the ventral spinal cord in the M-L direction and individually co-cultured with the ipsilateral CRN. After culture, immunostaining with anti-5-HT was used to confirm that descending serotonergic RST axons were growing from the CRN explants. (E) Decreasing M-L repulsion of serotonergic RST axons was detected clearly in the ventral spinal cord by the M-L graded assay. This graded repulsion was abolished effectively when the Shh antibody (5E1) or SANT-1 was added to the co-culture system or when Ptch $1^{\text {1loop } 2}$ was expressed in serotonergic RST axons by co-culturing the Ptch1 $1^{\Delta \text { Noop2 }}$-electroporated CRN with the explants from the ventral spinal cord. White arrowheads indicate deflected axons. White dashed lines outline the boundary of the explant from the ventral spinal cord (A, B and C). Scale bar: $200 \mu \mathrm{m}$. (F) Quantification of axon outgrowth in the M-L graded assay. Axon outgrowth was measured by the P/D ratio. M-L graded repulsion was abolished after the Shh signaling components were blocked individually. The experiment was replicated at least five times. Statistical analysis was performed using the Student's $t$-test. Results are presented as the mean \pm S.E.M. Control group: $0.376 \pm 0.03 ; 0.608 \pm 0.045 ; 0.801 \pm 0.047$; Shh antibody group: 0.897 $\pm 0.050 ; 0.899 \pm 0.039 ; 0.924 \pm 0.056 ;$ pCIG2-EGFP group: $0.335 \pm 0.049 ; 0.569 \pm 0.054 ; 0.808 \pm 0.058 ;$ Ptch $1^{\text {Lloop } 2}$ group: 0.953 $\pm 0.053 ; 0.949 \pm 0.070 ; 0.955 \pm 0.070$; DMSO group: $0.347 \pm 0.036 ; 0.562 \pm 0.040 ; 0.805 \pm 0.052$; SANT-1 group: $0.956 \pm 0.036$; $0.968 \pm 0.056 ; 0.979 \pm 0.044$. 
ventral spinal cord in the $\mathrm{M}-\mathrm{L}$ direction (A, B and $\mathrm{C}$ explants) and co-cultured each with the E12.5 CRN explant (Figure 5D). Whole-culture immunostaining with anti-5-HT was used to confirm that serotonergic RST axons were growing from the $\mathrm{CRN}$ explants. We found that the explants from the ventral spinal cord displayed an M-L graded repulsion toward serotonergic RST axons growing from the CRN explants, with the medial spinal cord displaying a stronger effect than the lateral spinal cord (Figure 5E). This graded repulsion provided further evidence that diffusible Shh from the floor plate establishes a high-to-low repulsive gradient in the M-L direction and restricts the descending serotonergic RST axons to the ventral and ventral-lateral funiculus. We used antibody 5E1, Ptch $1^{\text {4loop } 2}$ ex utero electroporation and SANT-1 to effectively block the M-L graded repulsion of Shh (Figure 5E). The P/D ratios of axon outgrowth differed significantly between experimental groups, which verified our hypothesis (Figure 5F). From the above data, we concluded that the repellent Shh guides the posterior projection of serotonergic RST axons in the A-P direction and simultaneously restricts these descending axons to the ventral and ventral-lateral funiculus in the M-L direction. The multidirectional repulsion of serotonergic RST axons is mediated by Shh binding to Ptch1 and subsequent derepression of Smo. Our results strongly suggested that Shh multidirectional repulsion is highly efficient and determines the spatial pathfinding of RST axons along the A-P and M-L directions in a concurrent and coordinated manner.

Shh signaling is required for the spatial pathfinding of serotonergic RST axons

Because the Shh signaling pathway plays several roles in determining the fate of various cells during development of the central nervous system, including serotonergic neurons in the brainstem, $S h h^{\text {loxp } / \text { - }}$ hypomorphic mice were used to analyze the role of Shh in axon guidance. According to the previous studies, the efficiency of Shh precursor processing was reduced in $S h h^{l o x p /-}$ mice due to the loxp sequence insertion flanking the processing domain. The $S h h^{\text {loxp } /}$ mice thus display hypomorphic activity, but no defects in cell differentiation or migration $[25,31]$. To exclude the possibility that the patterning of the spinal cord was affected by interference with Shh function, we used immunostaining for several markers to examine patterning in the E11.5 Shh $h^{\text {loxp }-}$ spinal cord. We did not observe any defect in spinal cord patterning after immunostaining for Pax6, NKX2.2, NKX6.1, Foxa2 or Olig2 (Supplementary information, Figure S8A). In addition, there was no obvious reduction in the number of serotonergic neurons in the E14.5 $S h h^{\text {loxp } /-}$ raphe nuclei (Supplementary information, Figure S9). These results indicated that we analyzed the phenotype in $S h h^{\text {loxp }}$ - mice under an unbiased condition. Whole-mount immunostaining with anti-5-HT showed that the length of serotonergic RST axons in the mutant mice was reduced greatly, and there were no obvious process defects when compared to the axon projection in wild-type (WT) mice (Figure 6B and 6C). Immunostaining of coronal sections with anti-5-HT showed a reduction in axon numbers in mutant mice (Figure 6B and 6C). This finding might reflect the fact that Shh downregulation may not affect the high-to-low Shh gradient required for the caudal projection of serotonergic RST axons, but it might make the gradient shorter or less steep and thus less efficient in guiding descending serotonergic RST axons over a long distance. To investigate this possibility, we examined Shh expression in the A-P and M-L directions of the E12.5 $S h h^{\text {loxp }-}$ spinal cord. The Shh gradient in the A-P direction became shorter and less steep in $S h h^{\text {loxp }-}$ mice compared to WT mice (Supplementary information, Figure S10A and $\mathrm{S} 10 \mathrm{~B}$ ). This finding indicated that the high-to-low repulsion gradient guiding serotonergic RST axons is dependent on the concentration of Shh, and the steepness and length of this gradient determine the efficiency of axon extension in the A-P direction. In addition, the concentration of Shh in the M-L direction was also reduced in $S h h^{\text {loxp } /-}$ mice compared to WT (Supplementary information, Figure S10C and S10D). However, the graded Shh distribution in the M-L direction still existed and is accordingly capable of restricting lesser amount of axons to the ventral and ventral-lateral funiculus.

Mice embryos electroporated in utero with Ptch $1^{4 l o o p 2}$ were used to analyze loss of Ptch1 function in vivo. Here, a constitutively active inhibitor of $P t c h 1^{4 l o o p 2}$ was expressed specifically in the region of the CRN. All Ptch $1^{\text {4loop } 2}$-expressing, serotonergic RST axons in the mutants were stalled at the upper cervical spinal cord and did not project into the lumbar spinal cord (Figure 7A and Supplementary information, Figure S1E). Misguided, Ptch $1^{\text {Sloop2 }}$-expressing serotonergic axons that looped back and had incorrect termination were also observed (Figure 7A). In coronal sections, a significant number of Ptch $1^{\text {4loop } 2}$-expressing serotonergic axons wandered to dorsal areas or to the midline (Figure 7A). The specific co-immunostaining pattern of EGFP and 5-HT in the E14.5 Ptch $1^{\text {4loop } 2}$-electroporated CRN is shown in highmagnification images in Supplementary information, Figure S11. The pathfinding errors of the Ptch1 ${ }^{\text {4loop2 }}$ expressing serotonergic RST axons were verified by quantification of axon pathfinding behavior (Figure 7B). These results indicated that an Shh repulsive signal 
A
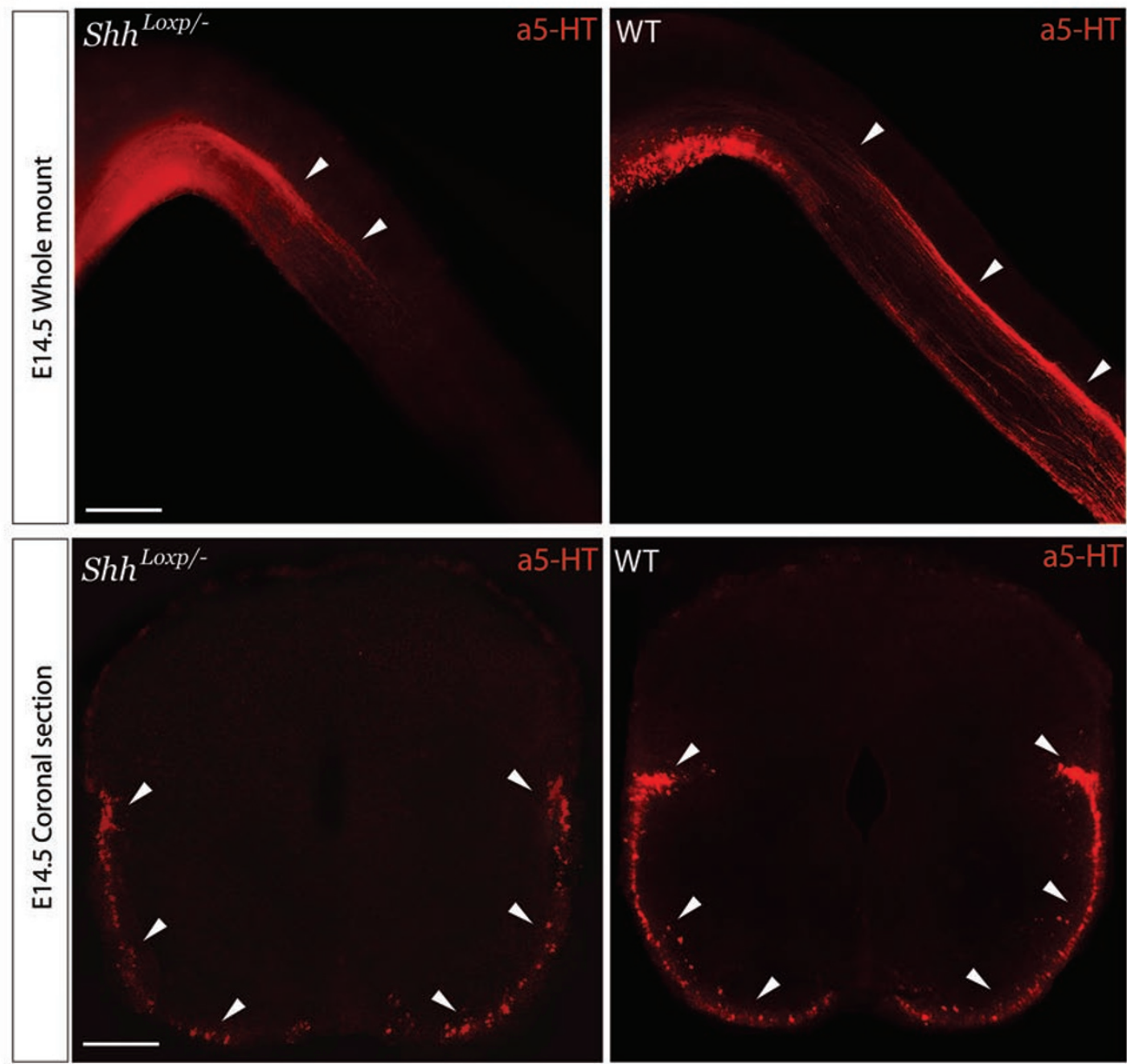

B
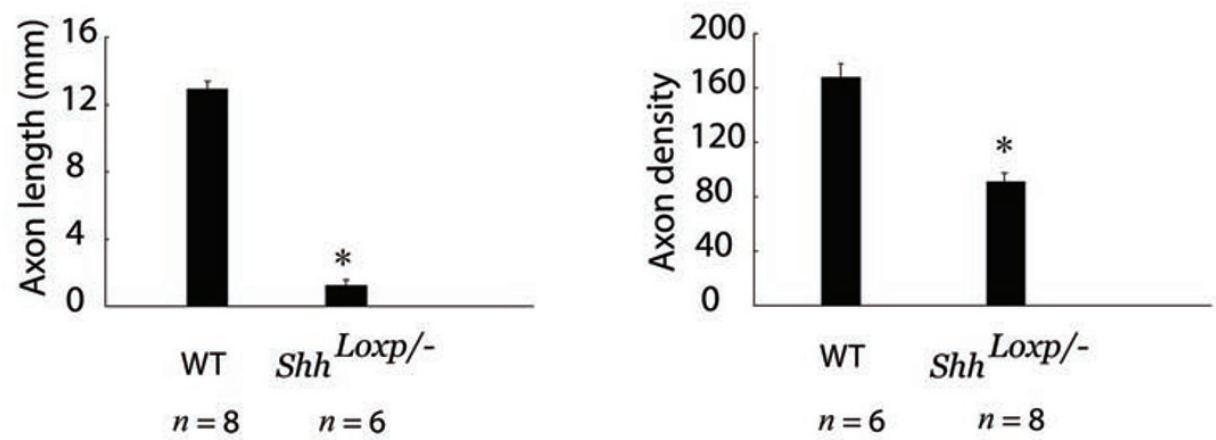

Figure 6 Shh is required for serotonergic RST axon pathfinding. (A) In E14.5 Sh/ ${ }^{\text {loxp/- }}$ hypomorphic mice, in which Shh expression was substantially downregulated, both axon length and the number of projecting serotonergic RST axons were greatly reduced compared to those in WT mice of the same age. Scale bar: $500 \mu \mathrm{m}$. White arrowheads indicate projecting serotonergic RST axons. (B) Quantification of the average length and density of serotonergic RST axons. Axon density was used as a measure of axon number. A significant reduction in the RST projection distance and density was observed in $S h h^{\text {loxp/- }}$ hypomorphic mice. Axon lengths $(\mathrm{mm})$ are presented as the mean \pm S.E.M. WT: $12.878 \pm 0.501 ;$ Sh ${ }^{\text {loxp } /-}: 1.236 \pm 0.333$.

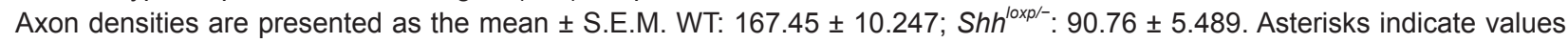
that differ significantly from those of the control groups $(P<0.05)$, and $n$ indicates the total number of embryos quantified. 
A



C



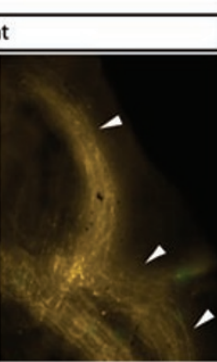

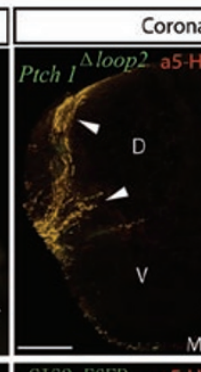

Coronal section
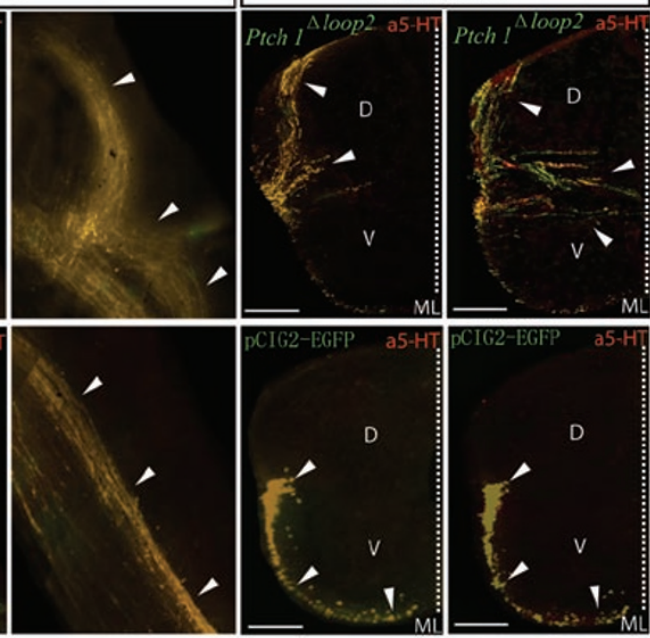

1.
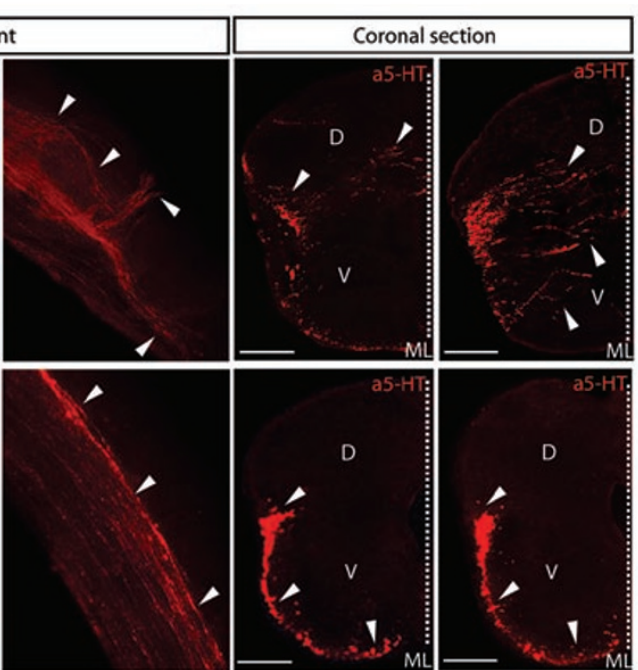

B
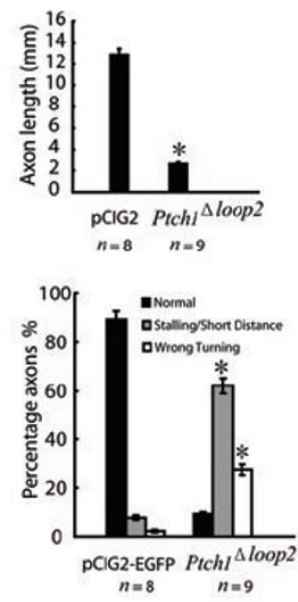

D
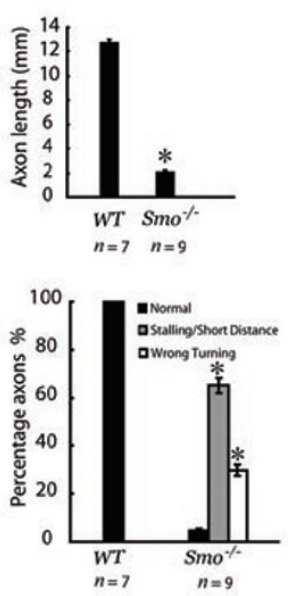

mediated by Shh-Ptch1 interactions is required to push the serotonergic RST axons caudally in the A-P direction and to restrict axons in the M-L direction to the ventral and ventral-lateral funiculus.

The Nestin-Cre; $\mathrm{Smo}^{-1-}$ mouse is one of several lines of Smo conditional knockout mice in which Smo is deleted specifically in the central nervous system beginning at E10.5 [27, 28]. The development of serotonergic neurons is not altered in these mutants $[32,33]$. To rule out the possibility that the patterning of the spinal cord was affected by the loss of Smo beginning at stage E10.5, we examined the patterning of the E11.5 Nestin-Cre; Smo ${ }^{-/-}$spinal cord by immunostaining for several markers. We did not detect any defect in patterning when mutant spinal cords were immunostained for Pax6, NKX2.2, NKX6.1, Foxa2 or Olig2 (Supplementary information, Figure S8B).
We also counted the number of serotonergic neurons in the E12.5 CRN in both mutant and WT mice, and analyzed the expression patterns of the genes encoding Lmx $1 b$ and Pet1 that critically control the development of serotonergic neurons in the brainstem [34-36]. We found no significant changes in the expression patterns of Lmx1b and Pet1 in serotonergic neurons of the caudal brainstem (Supplementary information, Figure S12B and S12D). Moreover, we also counted the number of cells immunoreactive for Lmx1b and 5-HT, Pet1 and 5-HT, and 5-HT in the caudal brainstem, finding no significant difference in cell numbers in mutant mice compared to WT mice (Supplementary information, Figure S12C and $\mathrm{S} 12 \mathrm{E}$ ). We can thus conclude that the phenotype of the Nestin-Cre; $\mathrm{Smo}^{-/-}$mice was not affected by loss of serotonergic neurons.

Whole-mount immunostaining (anti-5-HT) of E14.5 
Figure 7 Shh-Ptch1 interactions and Smo are required for mediating Shh-directed multidirectional repulsion. (A) Improper pathfinding of $P$ tch $1^{\Delta l o o p 2}$-expressing serotonergic RST axons after Ptch $1^{\Delta / o o p 2}$ in utero electroporation. After Ptch $1^{\Delta l o o p 2}$ was electroporated in utero into the CRN of E12.5 mice, most of Ptch $1^{\text {sloop2 }}$-expressing, serotonergic RST axons were stalled and only projected very short distances into the cervical spinal cord. By comparison, serotonergic RST axons in the control pCIG2-EGFPexpressing serotonergic RST axons projected as far as the lumbar level (also see Figure 1E). Some Ptch ${ }^{\Delta / \text { loop }^{2}}$-expressing serotonergic RST axons were significantly misguided and looped back in the anterior direction (white arrowheads). By comparison, pCIG2-EGFP-expressing serotonergic RST axons were tightly fasciculated and directionally uniform. Scale bar: $500 \mu \mathrm{m}$. In coronal sections, a bundle of Ptch $1^{\mathrm{Noop} 2}$-expressing serotonergic RST axons were misoriented to the dorsal area and midline of the spinal cord. Scale bar: $100 \mu \mathrm{m}$. (B) Quantification of serotonergic RST pathfinding behavior after pCIG2-EGFP or Ptch1 $1^{\text {Noop2 }}$ was electroporated in utero into the CRN. The top panel shows different projection distances of axons in pCIG2-EGFP- and Ptch $1^{\text {Nloop2}}$-electroporated mice. Data are presented as the mean \pm S.E.M. (mm). pCIG2-EGFP: $12.956 \pm 0.531 ;$ Ptch1 ${ }^{\text {Nloop2 }}: 2.603$ \pm 0.206 . The bottom panel shows the percentage of axons with normal or misguided behaviors in pCIG2-EGFP- and Ptch $1^{\text {1/oop2 }}$ electroporated mice. Data are presented as the mean \pm S.E.M. (\%). pCIG2-EGFP: $89.35 \pm 3.73 \%$ (normal projection); $8.21 \pm$ $1.01 \%$ (stalled or projected over a short distance); $2.44 \pm 0.67 \%$ (incorrectly turned). Ptch1 ${ }^{\text {\loop2 }}: 9.89 \pm 0.75 \%$ (normal projection); $62.21 \pm 3.07 \%$ (stalled or projected over a short distance); $27.9 \pm 2.23 \%$ (incorrectly turned). (C) Abnormal RST axon pathfinding in Nestin-Cre; Smo ${ }^{-/-}$mice. In E14.5 Nestin-Cre; Smo ${ }^{-1-}$ mice, the projection of serotonergic RST axons was obviously stalled in the upper cervical spinal cord. Some Smo-deficient serotonergic RST axons were disorientated with clear backward looping and wrong turns (white arrowheads). Scale bar: $500 \mu \mathrm{m}$. In coronal sections, many axons were misguided to the dorsal area (D) and wandered to the midline (ML). Scale bar: $100 \mu \mathrm{m}$. (D) Quantification of serotonergic RST pathfinding behavior in Nestin-Cre; $\mathrm{Smo}^{-1-}$ and WT mice. The top panel shows the different projection distances of serotonergic RST axons in NestinCre; $\mathrm{Smo}^{-/-}$and WT mice. Data are presented as the mean \pm S.E.M. (mm). WT: $12.744 \pm 0.356 ;$ Nestin-Cre; Smo ${ }^{-1-}: 2.087 \pm 0.211$. The bottom panel shows the percentage of axons with normal and misguided behaviors in Nestin-Cre; Smo ${ }^{-1-}$ and WT mice. Data are presented as the mean \pm S.E.M. (\%). WT: $100 \%$ normal; Nestin-Cre; Smo ${ }^{-1-}$ mice: $4.76 \pm 0.69 \%$ (normal projection); $65.33 \pm 2.99 \%$ (stalled or projected over a short distance); $29.81 \pm 2.41 \%$ (incorrectly turned). Error bars were derived from sets of experiments, and $n$ indicates the total number of embryos quantified. Asterisks indicate values that differ significantly from the values of the control groups $(P<0.05)$.

spinal cords from Nestin-Cre; Smo ${ }^{-/}$and WT mice showed that the majority of mutant serotonergic RST axons projected only into the cervical spinal cord, whereas WT serotonergic RST axons projected into the lumbar spinal cord (Figure 7C and Supplementary information, Figure S1E). As was the case with Ptch $1^{\text {4loop } 2}$-expressing serotonergic RST axons, stalled and misguided branches were observed at the end of the axonal projections in Nestin-Cre; Smo ${ }^{-/-}$mice. Some of the stalled serotonergic RST axons along the A-P axis in Nestin-Cre; $\mathrm{Smo}^{-/-}$mice were clearly misguided, and demonstrated looping back and incorrect turning when compared to the tight and directed projection observed in WT mice (Figure 7C). In coronal sections of mutant spinal cord, a significant number of axons diverged to dorsal areas or wandered to the midline (Figure 7C). The serotonergic RST axons in E14.5 Nestin-Cre; $\mathrm{Smo}^{-1-}$ mice were reduced in length and showed significantly misguided directionality (Figure 7D). These results strongly support the hypothesis that Shh repulsion not only directs serotonergic RST axons caudally but also restricts these axons to the ventral and ventral-lateral funiculus.

To further verify Shh repulsion of descending serotonergic axons, the Nestin-Cre; SmoM2 mouse was used as an Shh gain-of-function model. The Nestin-Cre; SmoM2 mouse is a transgenic mouse that constitutively expresses the active form of Smo and thereby increases Shh signaling, especially in the developing spinal cord [29]. In the Nestin-Cre; SmoM2 mouse, we found that serotonergic RST axonal projections were interrupted at the level of the upper cervical spinal cord (Supplementary information, Figures S13 and S1E). Most of these axons showed looping back and anterior deflection to the brainstem (Supplementary information, Figure S13). This finding indicated that enhanced Smo-mediated repulsion as a result of overexpression of the active form of Smo in the spinal cord damaged the graded Shh-Smo repulsion in the spinal cord. Stronger posterior repulsion pushed the descending serotonergic RST axons back to the brainstem. These results strongly suggested that Shh signaling repulsively guides the descending RST axons in the spinal cord. Moreover, Smo is required to activate this repulsion.

We concluded that the Shh signaling pathway mediates multidirectional repulsion and is necessary to push descending serotonergic RST axons in the A-P direction, and to simultaneously restrict these axons in the M-L direction to the ventral and ventral-lateral funiculus. This mechanism is used by serotonergic RST axons for spatial axon pathfinding in the A-P and M-L directions in the developing spinal cord. Thus, our discovery reveals a model of axon guidance in which one guidance cue concurrently and coordinately provides multidirectional 
axon guidance to establish accurate axon wiring in three dimensions.

\section{Discussion}

\section{Shh and Wnt may cooperate in A-P axon guidance}

Previous studies have found that Wnt family members control the ascending commissural axons and the descending corticospinal tract (CST) axons along the A-P axis of the spinal cord by interacting with different receptors, such as Frizzled family members or Ryk [4, $5,8,37]$. Shh signaling in the chicken directly repels anterior-projecting midline commissural axons by interacting with Hip and indirectly attracts these axons by regulating Wnt activity $[6,7]$. These results strongly suggest that Shh and Wnt cooperate in post-crossing commissural axon guidance. A recent study has shown that Wnt5a attracts the descending serotonergic axons along the A-P direction in the mouse brainstem [14]. In our study, we found that Shh signaling controlled the caudal projection of serotonergic RST axons by repelling axons toward the low expression region in the caudal spinal cord. These findings suggest that the descending serotonergic RST axons are guided initially by attractive Wnt signaling in the brainstem and subsequently by repulsive Shh signaling in the spinal cord. Although Shh or Wnt independently guide axons along the A-P axis of the spinal cord, additional cross-talk at the level of surface receptors or intracellular signaling molecules also may be important because previous studies have shown that there is signaling cross-talk between Wnt and Shh that regulates cell patterning and neurogenesis during development of the central nervous system [38, 39]. We cannot exclude the involvement of additional potential guidance cues in other descending and ascending axon pathways adjacent to the commissural ascending axons, CST and RST. Here, we have verified that the Shh signaling cascade is also involved in RST axon guidance along the A-P axis of the developing spinal cord. Future investigations will explore possible collaborative mechanisms between the Wnt and Shh signaling pathways in A-P axon guidance.

Shh repulsion requires Shh-Ptch interactions and derepression of Smo in the mouse

Hip-mediated Shh repulsion reportedly guides commissural axons ascending along the longitudinal axis in chicken [6]. However, we showed here that Shh repelled descending axons in the opposite direction in the mouse. It seems paradoxical that Shh would repel descending axons in the opposite direction in the mouse than in the chicken. To address this discrepancy, we explored the expression gradient of Shh in mouse E11.5 spinal cords, a stage at which the ascending commissural axons begin to project anteriorly and the Shh gradient is thought to be already established. We excluded any technical problems by determining the level of Shh mRNA expression in the spinal cord of chicks of the same age. However, we did not observe a clear Shh gradient along the A-P axis of the mouse, in contrast to the obvious increasing A-P gradient in embryonic chicken spinal cord (Supplementary information, Figure S14A and S14B). It should be noted that several descending nerve tracts are absent or follow different pathways in the chick when compared to the mouse. In addition, previous work has demonstrated Shh-dependent repulsion via derepression of Smo in developing retinal ganglion cell axons in mice [40-43]. Comparatively, Ptch and Smo are not involved in Shh repulsion of commissural axons in the chicken [6]. A reasonable explanation for these findings is that Shh-Hip interactions may repel ascending commissural axons uniquely in the developing chicken spinal cord and may not be relevant to the Shh repulsive signaling observed in the developing mouse spinal cord. Considering that commissural and RST axons are present and behave similarly in the two species, another plausible explanation is that the direction of the Shh gradient is time dependent because the projection of RST axons occurs much later than the projection of commissural axons in the mouse spinal cord. In mice, our studies and previous reports demonstrate Shh-dependent repulsion via derepression of Smo in developing retinal ganglion cell axons and spinal commissural axons [17, $18,44]$, which supports our contention that Shh repulsive signaling is mediated by Shh binding to Ptch1 and the subsequent derepression of Smo. In addition, the 5E1 antibody used in our culture assay can also block the binding of Shh to Hip [45]. Therefore, we cannot exclude Hip as a co-receptor with Ptch1 in our experiments. Future experiments must address these possibilities.

\section{Multidirectional axon guidance is better than monodirectional axon guidance}

Studies on axon guidance cues have shown that soluble axon guidance cues can diffuse in more than one dimension [46]. In addition, axons project and navigate through a three-dimensional space. Therefore, monodirectional axon guidance is not adequate to establish the precise projection pathway. Two possible hypotheses may account for multidirectional axon extension: (1) a combination of axon guidance molecules are used to drive axon pathfinding in three-dimensional space, with each molecule responsible for a single direction; or (2) one axon guidance molecule acts multidirectionally to establish the 
correct three-dimensional axon wiring. The present study convincingly demonstrates that the Shh signaling pathway is multidirectional because it simultaneously guided serotonergic RST axons in the A-P and M-L directions. The Shh signaling pathway also guides commissural axons in the dorso-ventral (D-V) direction [15]. We showed that one axon guidance molecule, Shh, concurrently drives serotonergic RST axons in multiple directions by repulsion down the descending tract, and toward the ventral and ventral-lateral funiculus.

An unidentified cue(s) may also play a role in positioning descending serotonergic axons in the spinal cord

Our study showed that Ptch1 $1^{\text {4loop2 }}$-expressing or Smodeficient RST axons were more dorsally restricted in the spinal cord than normal RST axons. Quantification of the axon positioning in the spinal cord (Supplementary information, Figure S15) showed that, among the misguided serotonergic axons, $68 \%$ of Ptch $1^{\text {4loop2 }}$ expressing axons wandered toward the midline and dorsal spinal cord, and $32 \%$ wandered toward the midline and stayed in the ventral spinal cord. In NestinCre; $\mathrm{Smo}^{-/-}$mice, we found that $61 \%$ of misguided serotonergic axons wandered toward the midline and dorsal cord, and $39 \%$ of misguided serotonergic axons wandered toward the midline and stayed in the ventral spinal cord. Therefore, we consider that another cue(s) affects axon positioning in the M-L or D-V direction, and it is very likely that the cue(s) is involved in positioning in the D-V direction. In particular, a cue(s) in the dorsal spinal cord may work together with Shh in the ventral spinal cord to determine the positioning of these axons. This result supports our speculation that Shh plays a role in the positioning of these axons, but another cue(s) in the dorsal spinal cord may also contribute to their positioning in the spinal cord.

An additional explanation is that Shh-Ptch-Smo signaling is required to keep the RST axons in the ventral half of the neural tube. It is also possible that higher concentrations of Shh at the midline repel the RST axons and that lower concentrations of Shh in the ventral-lateral spinal cord may attract the RST axons and keep them in that position, similar to the results found in an earlier study [42].

The Shh-mediated repulsive or attractive response is determined by co-receptors or downstream signaling molecules

The canonical Shh signaling pathway plays a pivotal role in cell-fate specification during development. The binding of Shh to Ptch and Boc/Cdo activates Smo, and leads to the activation of the Gli family of transcription factors. Gli-dependent transcription results in global changes in the cell [47-51]. As an attractive axon guidance molecule, Shh binds to its receptors, Boc and Ptch, and this activates Smo $[15,52]$. This then leads to the activation of the Src family kinase in commissural axons [16]. Previous work has shown a repulsive role for Shh in retinal ganglion cell axons during development [40-43]. In the chick spinal cord, the binding of Shh to Hip repels post-crossing commissural axons $[6,7]$. However, the components of the repulsive Shh signaling pathway have not been well identified in the mouse. In this study, we found in the mouse that Shh can also repulsively guide descending serotonergic RST axons in a Ptch1-Smo-dependent manner. This observation suggests that signaling downstream of Shh during axon guidance is more complicated than previously described. We suggest that Ptch and Smo mediate both the attractive and repulsive axon guidance of Shh and that a distinct surface co-receptor(s) or downstream molecule(s) may independently determine the outcome in different axons.

We also found that all axons behave in a similar way in $P t c h 1^{\text {4loop } 2}$-electroporated explants. Considering the efficiency of the ex utero electroporation, there should have been neurons that were not transfected with $P t c h 1^{4 l o o p 2}$ and thus should have responded to Shh axon guidance and behaved differently from transfected neurons. Recent studies on retinal axon growth reported that either cell-autonomous (retinal ganglion cell derived) Shh or non-cell-autonomous (midline derived) Shh appears to have a role in axon growth and guidance in retinal ganglion cells [17]. Therefore, we speculate that a non-cell-autonomous effect may contribute to the behavior of serotonergic axons in $P t c h 1^{\text {4loop } 2}$. electroporated explants. However, we need to further investigate the underlying molecular mechanisms of Shh axon guidance to clearly identify the switching mechanisms between Ptch- and Smo-dependent repulsion and attraction.

\section{Shh signaling directly guides serotonergic RST axons in} the developing spinal cord

Serotonergic axons from raphe nuclei in the brainstem descend to the spinal cord and ascend to various regions in the brain. The axons from several brainstem areas establish the serotonergic neural circuit in the central nervous system. Until recently, very little was known about the molecular mechanisms underlying the guidance of descending serotonergic axons from the RST, which is considered to be an inhibitory pathway involved in pain, sensory and motor functions. The serotonergic RST can be assessed in an anterograde fashion using simple immunohistology because the RST is the only source of serotonergic input to the developing spinal cord. 
In our study of Ptch1 and Smo mutants, we observed fatal axon pathfinding errors of serotonergic RST axons as they entered the spinal cord, demonstrating that Shh signaling is the primary cue that maintains spatial axon pathfinding for RST axons after they leave the brainstem. Despite these axon guidance defects, we observed that some serotonergic RST axons continued to descend a very short distance along the spinal A-P axis. We hypothesize that when these axons sprout from serotonergic neurons in the brainstem, they are able to extend a short distance before finding axon guidance cues in the spinal cord. A recent study showed that Wnt-Frizzled attractive axon guidance signaling underlies the extension of these axons in the brainstem [14]. However, this extension cannot continue in the spinal cord if the growth cones are unable to detect the appropriate Shh gradient. In this case, axons may stall or become disorientated in response to gradients of inappropriate cues. Indeed, after Smo deletion or Ptch $1^{\text {4loop2 }}$ expression, we observed serotonergic RST axons in the A-P direction projecting for short distances, with some axons looping back. Similarly, along the M-L direction, we observed that some axons were misguided to the midline and dorsal area after Smo deletion or $P t c h 1^{\text {Aloop } 2}$ expression. We speculate that all of these errors in serotonergic RST axon pathfinding in Shh signaling-mutant mice are due to the inability to detect Shh signaling in both the A-P and M-L directions. This strongly supports the idea that Shh signaling is the direct axon guidance signaling system for the spatial projection of serotonergic RST axons in the spinal cord. However, we cannot exclude the possibility that another cue(s) (e.g., Wnt family members) also exists in the spinal cord and may play a collaborative role in the spatial pathfinding of serotonergic RST axons. In future investigations, we will explore whether Shh and Wnt cooperate to guide longitudinal axons in the mouse spinal cord and which mechanisms underlie their collaboration.

\section{Materials and Methods}

\section{Animals and treatments}

All animal experiments conformed to the regulations of the Animal Research Committee of the Institute of Biophysics, Chinese Academy of Sciences; these regulations are based on the US National Institute of Health (NIH) Guidelines for the Use and Care of Laboratory Animals. CD-1 and C57BL6/J mice were obtained from Vital River Inc. (Beijing, China).

\section{Labeling the trajectory of developing serotonergic RST ax- ons}

Whole brains and spinal cords were dissected from E11.5 to E16.5 embryos and fixed in 4\% paraformaldehyde (PFA). Immunostaining of whole mounts and transverse sections of spinal cords was performed by incubating samples with a polyclonal antibody against 5-HT (rabbit IgG; diluted 1:500; Sigma) and then with a Cy3-conjugated goat anti-rabbit IgG secondary antibody (1:500; Jackson Laboratory). Immunohistochemistry was carried out as described [53,54].

\section{Analysis of Shh expression gradients in developing spinal cords}

A 580-bp cDNA fragment at the 3' end of the mouse Shh coding region was amplified by $\mathrm{PCR}$ using the forward primer, $5^{\prime}$ ACTTCCTCACCTTCCTGGAC- 3 ', and the reverse primer, 5'CCCAAGGGATGCATGGTCTC-3', and then cloned into vector pGMT (TaKaRa Biotech). Sense and antisense RNA probes for Shh were synthesized and labeled with a DIG RNA labeling kit (Roche). Embryonic spinal cords from stages E11.5 to E14.5 were dissected, fixed in 4\% PFA and embedded in Tissue-Tek O.C.T. (Electron Microscope Science). Coronal sections $(20 \mu \mathrm{m})$ were cryosectioned and collected along the A-P axis at $800-\mu \mathrm{m}$ intervals or $20-\mu \mathrm{m}$ thick horizontal sections were cryosectioned from the anterior to the posterior spinal cord. In situ hybridization was conducted as described [55]. Immunostaining for Shh was conducted using a polyclonal antibody against Shh (rabbit IgG; diluted 1:100; Santa Cruz Biotechnology) as described [31, 56]. The relative immunostaining intensity was analyzed using Image J software (NIH) and averaged from at least five replicate sets. Statistical analysis was performed using Student's $t$-test. All values are represented as mean \pm S.E.M.

Analysis of Ptch1 and Smo expression patterns in embryonic CRN and projecting RST axons

cDNA fragments of the mouse Ptch1 and Smo coding regions were amplified by PCR $[29,57]$ and then cloned into pGMT. Sense and antisense RNA probes for Ptchl and Smo were synthesized, and labeled with a DIG RNA labeling kit. Embryonic brainstems and spinal cords were dissected from mouse embryos and fixed in 4\% PFA, and the CRN and pioneer serotonergic RST axons then were dissected from the tissue. Sagittal sections of $10-\mu \mathrm{m}$ thickness were prepared (Figure 2A). Coronal sections of embryonic spinal cords containing serotonergic RST axons were also prepared (Figure 2F). In situ hybridization was conducted as described [55]. Co-immunostaining was conducted using a monoclonal antibody against Ptch1 (mouse IgG; 1:100; R\&D) or a polyclonal antibody against Smo (rabbit IgG; 1:100; Santa Cruz Biotechnology) together with a polyclonal antibody against 5-HT (goat IgG; 1:500; ImmunoStar). Negative controls consisted of preincubation of anti-Ptch1 with $10 \mu \mathrm{g} / \mathrm{ml}$ of recombinant Ptch1 protein or staining of tissue from a Nestin-Cre; $\mathrm{Smo}^{-1-}$ mouse. Secondary antibodies (Cy2-conjugated goat anti-rabbit IgG or Cy2-conjugated goat anti-mouse $\operatorname{IgG}$ with $\mathrm{Cy} 3$-conjugated rabbit anti-goat IgG; 1:500) were obtained from the Jackson Laboratory. Immunohistochemistry was carried out as described [53, 54]. Pairs of digital images were merged after collection using a fluorescence microscope.

\section{CRN explant assay}

E12.5 mice embryos were sacrificed and placed in L15 medium (Gibco). Under a dissecting microscope, the whole brainstem and spinal cord were teased out using fine forceps while immersed in ice-cold L15. Unwanted portions of the brainstem were trimmed away, and ipsilateral CRN explants were dissected after identifying 
the CRN using whole-mount immunostaining with anti-5-HT (Figure 3A). In most cases, only three explants were dissected from one ipsilateral CRN to guarantee the accuracy of tissue collection.

In the cell aggregate assay, COS1 cells were transfected with an Shh cDNA construct using FuGene 6 transfection reagent (Roche). COS1 cells that were transfected with empty expression vectors were used as controls [4, 5]. Immunostaining with anti-Shh (1:100; Santa Cruz Biotechnology) was conducted to examine Shh expression in transfected COS1 cells after overnight culture. Shhexpressing COS1 cells were aggregated as we have described [5]. The first layer of rat collagen gel was prepared and E12.5 CRN explants were dissected as described above (Figure 3A). COS1 cell aggregates and explants were transferred onto the polymerized collagen layer, with the explants facing the cell aggregates. A secondary collagen layer was placed on top of them to form an encasing collagen matrix.

In the A-P graded assay, using fine forceps and razor blade knives, each spinal cord was cut into two identical halves from the upper cervical to the thoracic level along the midline, like an open book, and trimmed to obtain 0.5 - $\mathrm{cm}$-long pieces (Figure 4A). Trimmed spinal cord samples were transferred onto dishes coated with a collagen gel layer, with the midline side of the spinal cord facing the surface of the collagen. Three ipsilateral CRN explants were placed in the A-P direction toward the ventral side of the spinal cord (Figure 4A). An additional layer of collagen was then added and polymerized at $37^{\circ} \mathrm{C}$.

For the M-L co-culture assay, E12.5 cervical spinal cords were cut transversely into 0.1 - to 0.2 -mm-thick slices, and the dorsal part was discarded (Supplementary information, Figure S7A). Slices of the ventral spinal cord and the CRN explants were stored temporarily in ice-cold L15. Slices of the ventral spinal cord and the CRN explants were then transferred onto dishes coated with a collagen gel layer, and each CRN explant was oriented toward the lateral side of the ventral spinal cord slice (Supplementary information, Figure S7A).

In the M-L graded assay, three explants from the E12.5 ventral cord in the M-L direction were dissected (A, B and C explants shown in Figure 5B and 5D) and immediately transferred onto collagen-coated dishes. Afterward, each explant was co-cultured individually with one ipsilateral CRN explant (Figure 5D).

After complete polymerization at $37^{\circ} \mathrm{C}$, all explants were cultured for $60 \mathrm{~h}$ in $0.5 \mathrm{ml}$ of MEM supplemented with $10 \%$ fetal bovine serum. Culture growth was terminated by the addition of 4\% PFA. Immunostaining was conducted with polyclonal anti-5HT (1:500; Sigma) and the Cy3-conjugated goat anti-rabbit IgG secondary antibody (1:500) to label the growing serotonergic axons from the CRN.

Images were taken under fluorescence microscope. The total length of the axons emerging from explants was measured using Image J software (NIH). Quantification was based on the P/D ratio, which was defined as the ratio of axon length in the proximal quadrant to that in the distal quadrant. The experiment was repeated at least five times. The results are presented as mean \pm S.E.M. and statistical significance was determined using Student's $t$-test [5].

\section{In vitro interference with the Shh signaling cascade}

To block Shh activity, an antibody against Shh (5E1; DSHB) [18,
58] was added to the culture medium at a final concentration of $50 \mathrm{ng} / \mathrm{ml}$ and the vehicle for the antibody was used as a negative control. To block Smo activity, the antagonist SANT-1 (Sigma) [16] was added to the culture medium at a final concentration of 50 $\mu \mathrm{g} / \mathrm{ml}$, and the vehicle, dimethylsulfoxide (DMSO) was used as a negative control.

To block Ptch1 activity, Ptch $1^{\text {Sloop } 2}$ Myc-IRES-EGFP or pCIG2IRES-EGFP DNA $[17,23](1 \mu \mathrm{g} / \mu \mathrm{l}$ with $0.05 \%$ fast blue in PBS $)$ was electroporated ex utero into the $\mathrm{CRN}$ of E12.5 mouse embryos using an electroporator (ECM 830; BTX) and 5-mm forcep electrodes (CUY650-5; Protech International). Electroporation was performed by positioning the brainstem of each embryo between the forcep electrodes and administering 5 electric pulses $(40 \mathrm{~V}, 100$ ms) at 1 -s intervals $[59,60]$. CRN explants were micro-dissected after electroporation and immediately transferred to the culture assay. The culture medium was replenished daily. To identify Ptch $1^{\text {4loop } 2}$-expressing serotonergic axons growing from the CRN, co-immunostaining with anti-GFP (mouse IgG; $1: 1000$; Sigma) and primary antibody against 5-HT (rabbit IgG; $1: 500$; Sigma) was conducted. Immunostaining and quantification was conducted as described $[53,54]$.

\section{In utero electroporation}

Pregnant 12.5-day C57BL6/J females were anesthetized by intraperitoneal injection of sodium pentobarbital $(0.625 \mathrm{mg}$ per 10 g body weight). The abdomen was opened and the uterine horns were exposed. The $P t c h 1^{\text {4loop } 2}-M y c$-IRES-EGFP or pCIG2-IRESEGFP DNA solution $(2 \mu \mathrm{g} / \mu 1$ with $0.05 \%$ fast blue in PBS) was injected into the CRN of each embryo using a graduated, pulledglass micropipette. The junction part of the hindbrain and the spinal cord of each embryo was placed between forcep electrodes (CUY650-5; Protech International) and 5 square electric pulses $(40 \mathrm{~V}, 100 \mathrm{~ms})$ were passed at 1 -s intervals using an electroporator (ECM 830; BTX) $[59,60]$. The wall and skin of the abdominal cavity were sutured and closed, and the embryos were allowed to develop normally until E16.5.

\section{Analysis of mutants}

$\mathrm{Shh}^{\text {loxp/-}}, \mathrm{Ptch}^{\text {4loop2 }}$-electroporated, Nestin-Cre; $\mathrm{Smo}^{-/-}$and Nestin-Cre; SmoM2 mutants were analyzed. E14.5 mutant and control embryos were sacrificed and fixed in 4\% PFA overnight. Whole brains and spinal cords were dissected from fixed embryos. For Shh ${ }^{\text {loxp }-}$, Nestin-Cre; $\mathrm{Smo}^{-/}$and Nestin-Cre; SmoM2 mutants, whole-mount immunostaining with anti-5-HT (1:500; Sigma) was conducted for 3 days at $4{ }^{\circ} \mathrm{C}$, followed by immunostaining with Cy3-conjugated goat anti-rabbit IgG (1:500) overnight at $4{ }^{\circ} \mathrm{C}$. For Ptch $1^{4 l o o p 2}$-electroporated mutants, whole-mount coimmunostaining with anti-5-HT (1:500; Sigma) and anti-GFP (1:500; Sigma) was conducted for 3 days at $4{ }^{\circ} \mathrm{C}$, followed by immunostaining with Cy3-conjugated goat anti-rabbit IgG (1:500) and Cy2-conjugated goat anti-mouse $\operatorname{IgG}$ (1:500) overnight at $4{ }^{\circ} \mathrm{C}$. After washing with PBS, the whole-mount brainstems and spinal cords were transferred to slides, covered gently with coverslips and then observed under a fluorescence microscope. Images were collected and quantification was performed using Image J software (NIH). The length of descending serotonergic RST axons and the percentage of axon pathfinding errors were determined. The length of each group and the percentage of axon pathfinding errors were averaged from at least five replicate sets 
of different E14.5 spinal cords. Statistical analysis was performed using Student's $t$-test. All values are presented as mean \pm S.E.M.

To ensure that our analysis was based on an unbiased system, we also examined the patterning of E11.5 spinal cord in $S h h^{\text {loxp } /-}$ and Nestin-Cre; $\mathrm{Smo}^{-/}$mice by immunostaining with anti-Pax-6 (Rabbit IgG; 1:300; MBL), anti-Foxa2 (mouse IgG; 1:50; DSHB), antiNKX2.2 (mouse IgG; 1:50; DSHB), anti-NKX6.1 (mouse IgG1; 1:100; DSHB) and anti-Olig2 (Rabbit IgG; 1:200; Millipore). Immunostaining was conducted using coronal sections from E11.5 spinal cord. The secondary antibody was Cy2-conjugated antimouse IgG (1:500) or Cy2-conjugated anti-rabbit IgG (1:500). Immunohistochemistry was conducted as described [55].

To confirm that the observed axon pathfinding errors were not confounded with serotonergic cell development in the CRN of Nestin-Cre; $\mathrm{Smo}^{-/-}$mutants, co-immunostaining with anti-Lmx1b (rabbit IgG; 1:500) generated in Ding Lab [61] and anti-5-HT (goat IgG; 1:500; ImmunoStar), or anti-Pet1 (rabbit IgG; 1:500; Santa Cruz Biotechnology) and anti-5-HT was conducted using horizontal sections from the caudal brainstem, which contained serotonergic CRN neurons (Supplementary information, Figure S12A). Secondary antibodies were Cy2-conjugated anti-rabbit $\operatorname{IgG}(1: 500)$ with Cy3-conjugated anti-goat IgG (1:500). Immunohistochemistry was conducted as described [55]. Serial sections containing positive signals were taken pictures under fluorescence microscope. All pairs of digital images were collected and merged. The number of different immunoreactive neurons in the E14.5 caudal brainstem was counted stereologically in a physical disector using a fractionator paradigm as described [54]. In addition, we counted numbers of serotonergic neurons in the brainstem of E14.5 Shh $h^{\text {loxp }-}$ mice using the same methodology.

\section{Acknowledgments}

We greatly appreciate the support of Dr Shigang He (Institute of Biophyscis, Chinese Academy of Sciences, China) for facilitating this research. We also appreciate the generous gift of different Shh and Smo mutants from Drs Chin Chiang and Xi Huang (Vanderbilt University Medical Center, USA), the Ptch1 $1^{\text {4loop2 }}$ construct from Dr Paola Bovolenta (Instituto Cajal (CSIC) and CIBER de Enfermedades Raras (CIBERER), Spain), the Lmx1b antibody from Dr Yuqian Ding (Tongji University, China) and the Pax6 antibody from Dr Zhenggang Yang (Fudan University, China). We thank Dr Helen Cooper (University of Queensland, Australia) for critical reading of the manuscript. We thank Drs Javid A Dar and Junkui Ai (University of Pittsburgh, USA) for proofreading the manuscript. This study was supported by grants from the $\mathrm{Na}-$ tional Natural Science Foundation of China (30640006, 30771063, 30871425 and 30990261) and the Chinese Academy of Sciences (KSCX2-EW-Q-11).

\section{References}

1 Tessier-Lavigne M, Goodman CS. The molecular biology of axon guidance. Science 1996; 274:1123-1133.

2 Dickson BJ. Molecular mechanisms of axon guidance. Science 2002; 298:1959-1964.

3 Tessier-Lavigne M. Axon guidance by molecular gradients. Curr Opin Neurobiol 1992; 2:60-65.
4 Lyuksyutova AI, Lu CC, Milanesio N, et al. Anterior-posterior guidance of commissural axons by Wnt-frizzled signaling. Science 2003; 302:1984-1988.

5 Liu Y, Shi J, Lu CC, et al. Ryk-mediated Wnt repulsion regulates posterior-directed growth of corticospinal tract. Nat Neurosci 2005; 8:1151-1159.

6 Bourikas D, Pekarik V, Baeriswyl T, et al. Sonic hedgehog guides commissural axons along the longitudinal axis of the spinal cord. Nat Neurosci 2005; 8:297-304.

7 Domanitskaya E, Wacker A, Mauti O, et al. Sonic hedgehog guides post-crossing commissural axons both directly and indirectly by regulating Wnt activity. J Neurosci 2010 ; 30:11167-11176.

8 Zou Y. Navigating the anterior-posterior axis with Wnts. Neuron 2006; 49:787-789.

9 Stoeckli ET. Longitudinal axon guidance. Curr Opin Neurobiol 2006; 16:35-39.

10 Liu ZY, Zhuang DB, Lunderberg T, Yu LC. Involvement of 5-hydroxytryptamine(1A) receptors in the descending antinociceptive pathway from periaqueductal gray to the spinal dorsal horn in intact rats, rats with nerve injury and rats with inflammation. Neuroscience 2002; 112:399-407.

11 Deumens R, Koopmans GC, Joosten EA. Regeneration of descending axon tracts after spinal cord injury. Prog Neurobiol 2005; 77:57-89.

12 Jones SL, Light AR. Termination patterns of serotoninergic medullary raphespinal fibers in the rat lumbar spinal cord: an anterograde immunohistochemical study. J Comp Neurol 1990; 297:267-282.

13 Jones SL, Light AR. Serotoninergic medullary raphespinal projection to the lumbar spinal cord in the rat: a retrograde immunohistochemical study. J Comp Neurol 1992; 322:599610 .

14 Fenstermaker AG, Prasad AA, Bechara A, et al. Wnt/ Planar cell polarity signaling controls the anterior-posterior organization of monoaminergic axons in the brainstem. $J$ Neurosci 2010; 30:16053-16064.

15 Charron F, Stein E, Jeong J, McMahon AP, Tessier-Lavigne M. The morphogen sonic hedgehog is an axonal chemoattractant that collaborates with netrin-1 in midline axon guidance. Cell 2003; 113:11-23.

16 Yam PT, Langlois SD, Morin S, Charron F. Sonic hedgehog guides axons through a noncanonical, Src-family-kinasedependent signaling pathway. Neuron 2009; 62:349-362.

17 Sanchez-Camacho C, Bovolenta P. Autonomous and nonautonomous Shh signalling mediate the in vivo growth and guidance of mouse retinal ganglion cell axons. Development 2008; 135:3531-3541.

18 Parra LM, Zou Y. Sonic hedgehog induces response of commissural axons to Semaphorin repulsion during midline crossing. Nat Neurosci 2009; 13:29-35.

19 Sanchez-Camacho C, Bovolenta P. Emerging mechanisms in morphogen-mediated axon guidance. Bioessays 2009; 31:1013-1025.

20 Briscoe J. Making a grade: Sonic Hedgehog signalling and the control of neural cell fate. EMBO J 2009; 28:457-465.

21 Salinas PC. The morphogen sonic hedgehog collaborates with netrin-1 to guide axons in the spinal cord. Trends Neurosci 2003; 26:641-643. 
22 Huangfu D, Anderson KV. Signaling from Smo to Ci/Gli: conservation and divergence of Hedgehog pathways from Drosophila to vertebrates. Development 2006; 133:3-14.

23 Briscoe J, Chen Y, Jessell TM, Struhl G. A hedgehoginsensitive form of patched provides evidence for direct longrange morphogen activity of sonic hedgehog in the neural tube. Mol Cell 2001; 7:1279-1291.

24 Chiang C, Litingtung Y, Lee E, et al. Cyclopia and defective axial patterning in mice lacking Sonic hedgehog gene function. Nature 1996; 383:407-413.

25 Huang X, Litingtung Y, Chiang C. Region-specific requirement for cholesterol modification of sonic hedgehog in patterning the telencephalon and spinal cord. Development 2007; 134:2095-2105.

26 Lewis PM, Dunn MP, McMahon JA, et al. Cholesterol modification of sonic hedgehog is required for long-range signaling activity and effective modulation of signaling by Ptc1. Cell 2001; 105:599-612.

27 Long F, Zhang XM, Karp S, Yang Y, McMahon AP. Genetic manipulation of hedgehog signaling in the endochondral skeleton reveals a direct role in the regulation of chondrocyte proliferation. Development 2001; 128:5099-5108.

28 Tronche F, Kellendonk C, Kretz O, et al. Disruption of the glucocorticoid receptor gene in the nervous system results in reduced anxiety. Nat Genet 1999; 23:99-103.

29 Goodrich LV, Johnson RL, Milenkovic L, McMahon JA, Scott MP. Conservation of the hedgehog/patched signaling pathway from flies to mice: induction of a mouse patched gene by Hedgehog. Genes Dev 1996; 10:301-312.

30 Soula C, Danesin C, Kan P, Grob M, Poncet C, Cochard P. Distinct sites of origin of oligodendrocytes and somatic motoneurons in the chick spinal cord: oligodendrocytes arise from Nkx2.2-expressing progenitors by a Shh-dependent mechanism. Development 2001; 128:1369-1379.

31 Li Y, Zhang H, Litingtung Y, Chiang C. Cholesterol modification restricts the spread of Shh gradient in the limb bud. Proc Natl Acad Sci USA 2006; 103:6548-6553.

32 Craven SE, Lim KC, Ye W, Engel JD, de Sauvage F, Rosenthal A. Gata2 specifies serotonergic neurons downstream of sonic hedgehog. Development 2004; 131:1165-1173.

33 Ye W, Shimamura K, Rubenstein JL, Hynes MA, Rosenthal A. FGF and Shh signals control dopaminergic and serotonergic cell fate in the anterior neural plate. Cell 1998; 93:755-766.

34 Cheng L, Chen CL, Luo P, et al. Lmx1b, Pet-1, and Nkx2.2 coordinately specify serotonergic neurotransmitter phenotype. J Neurosci 2003; 23:9961-9967.

35 Ding YQ, Marklund U, Yuan W, et al. Lmx1b is essential for the development of serotonergic neurons. Nat Neurosci 2003; 6:933-938.

36 Zhao ZQ, Scott M, Chiechio S, et al. Lmx1b is required for maintenance of central serotonergic neurons and mice lacking central serotonergic system exhibit normal locomotor activity. J Neurosci 2006; 26:12781-12788.

37 Schmitt AM, Shi J, Wolf AM, Lu CC, King LA, Zou Y. Wnt-Ryk signalling mediates medial-lateral retinotectal topographic mapping. Nature 2006; 439:31-37.

38 Alvarez-Medina R, Cayuso J, Okubo T, Takada S, Marti E. Wnt canonical pathway restricts graded Shh/Gli patterning activity through the regulation of Gli3 expression.
Development 2008; 135:237-247.

39 Joksimovic M, Yun BA, Kittappa R, et al. Wnt antagonism of Shh facilitates midbrain floor plate neurogenesis. Nat Neurosci 2009; 12:125-131.

40 Trousse F, Marti E, Gruss P, Torres M, Bovolenta P. Control of retinal ganglion cell axon growth: a new role for Sonic hedgehog. Development 2001; 128:3927-3936.

41 Gordon L, Mansh M, Kinsman H, Morris AR. Xenopus sonic hedgehog guides retinal axons along the optic tract. Dev Dyn 2010; 239:2921-2932.

42 Kolpak A, Zhang J, Bao ZZ. Sonic hedgehog has a dual effect on the growth of retinal ganglion axons depending on its concentration. J Neurosci 2005; 25:3432-3441.

43 Kolpak AL, Jiang J, Guo D, et al. Negative guidance factorinduced macropinocytosis in the growth cone plays a critical role in repulsive axon turning. $J$ Neurosci 2009; 29:1048810498.

44 Fabre PJ, Shimogori T, Charron F. Segregation of ipsilateral retinal ganglion cell axons at the optic chiasm requires the Shh receptor Boc. J Neurosci 2010; 30:266-275.

45 Maun HR, Wen X, Lingel A, et al. Hedgehog pathway antagonist 5E1 binds hedgehog at the pseudo-active site. $J$ Biol Chem 2010; 285:26570-26580.

46 Dickson BJ, Gilestro GF. Regulation of commissural axon pathfinding by slit and its Robo receptors. Annu Rev Cell Dev Biol 2006; 22:651-675.

47 Dessaud E, McMahon AP, Briscoe J. Pattern formation in the vertebrate neural tube: a sonic hedgehog morphogen-regulated transcriptional network. Development 2008; 135:2489-2503.

48 Stone DM, Hynes M, Armanini M, et al. The tumoursuppressor gene patched encodes a candidate receptor for Sonic hedgehog. Nature 1996; 384:129-134.

49 Alcedo J, Ayzenzon M, Von Ohlen T, Noll M, Hooper JE. The Drosophila smoothened gene encodes a seven-pass membrane protein, a putative receptor for the hedgehog signal. Cell 1996; 86:221-232.

50 van den Heuvel M, Ingham PW. Smoothened encodes a receptor-like serpentine protein required for hedgehog signalling. Nature 1996; 382:547-551.

51 Murone M, Rosenthal A, de Sauvage FJ. Sonic hedgehog signaling by the patched-smoothened receptor complex. Curr Biol 1999; 9:76-84.

52 Okada A, Charron F, Morin S, et al. Boc is a receptor for sonic hedgehog in the guidance of commissural axons. Nature 2006; 444:369-373.

53 Liu Y, Wang X, Lu CC, et al. Repulsive Wnt signaling inhibits axon regeneration after CNS injury. J Neurosci 2008; 28:8376-8382.

54 Li X, Li YH, Yu S, Liu Y. Upregulation of Ryk expression in rat dorsal root ganglia after peripheral nerve injury. Brain Res Bull 2008; 77:178-184.

55 Frohman MA, Boyle M, Martin GR. Isolation of the mouse Hox-2.9 gene; analysis of embryonic expression suggests that positional information along the anterior-posterior axis is specified by mesoderm. Development 1990; 110:589-607.

56 Thayer SP, di Magliano MP, Heiser PW, et al. Hedgehog is an early and late mediator of pancreatic cancer tumorigenesis. Nature 2003; 425:851-856.

57 Rice R, Spencer-Dene B, Connor EC, et al. Disruption 
of Fgf10/Fgfr2b-coordinated epithelial-mesenchymal interactions causes cleft palate. J Clin Invest 2004; 113:16921700.

58 Ericson J, Morton S, Kawakami A, Roelink H, Jessell TM. Two critical periods of Sonic Hedgehog signaling required for the specification of motor neuron identity. Cell 1996; 87:661673.

59 de Nijs L, Leon C, Nguyen L, et al. EFHC1 interacts with microtubules to regulate cell division and cortical development. Nat Neurosci 2009; 12:1266-1274.

60 Harvey AR, Ehlert E, de Wit J, et al. Use of GFP to analyze morphology, connectivity, and function of cells in the central nervous system. Methods Mol Biol 2009; 515:63-95.

61 Dai JX, Hu ZL, Shi M, Guo C, Ding YQ. Postnatal ontogeny of the transcription factor Lmx $1 \mathrm{~b}$ in the mouse central nervous system. J Comp Neurol 2008; 509:341-355.

(Supplementary information is linked to the online version of the paper on the Cell Research website.) 